\title{
TRAVELING WAVE SOLUTIONS \\ OF DIFFUSIVE LOTKA-VOLTERRA EQUATIONS: A HETEROCLINIC CONNECTION IN $\mathbf{R}^{4}$
}

\author{
BY
}

STEVEN R. DUNBAR

\begin{abstract}
We establish the existence of traveling wave solutions for a reaction-diffusion system based on the Lotka-Volterra differential equation model of a predator and prey interaction. The waves are of transition front type, analogous to the solutions discussed by Fisher and Kolmogorov et al. for a scalar reaction-diffusion equation. The waves discussed here are not necessarily monotone. There is a speed $c^{*}>0$ such that for $c>c^{*}$ there is a traveling wave moving with speed $c$. The proof uses a shooting argument based on the nonequivalence of a simply connected region and a nonsimply connected region together with a Liapunov function to guarantee the existence of the traveling wave solution. The traveling wave solution is equivalent to a heteroclinic orbit in 4-dimensional phase space.
\end{abstract}

1. Introduction. The purpose of this paper is to prove the existence of traveling wave solutions of the reaction-diffusion system

$$
u_{t}=D_{1} u_{x x}+A u(1-u / K)-B u w, \quad w_{t}=D_{2} w_{x x}-C w+E u w .
$$

The reaction terms are the familiar Lotka-Volterra growth terms with a logistic (or Verhulst) growth limitation on the prey species. Here $u(t, x)$ and $w(t, x)$ are the densities of the prey and predator species at time $t$ and position $x \in \mathbf{R}^{1}$. The traveling waves found are of transition front type analogous to the front solutions discussed by Fisher [10] and Kolmogorov et al. [17]. However, the waves found in this paper are not necessarily monotone. There is a value $c^{*}>0$ such that for $c>c^{*}$ there is a traveling wave solution of system (1) moving with speed $c$. See Theorem 1 in $\$ 2$ for the precise statement of the result. For simplicity we consider only one space dimension.

The technique used to establish the existence of the traveling waves is a shooting argument in $\mathbf{R}^{4}$, together with a Liapunov function and LaSalle's Invariance Principle. The shooting argument is unusual in that the existence of a traveling wave solution is guaranteed by the nonequivalence of a simply connected region and a punctured disk, rather than the nonequivalence of an interval and a disconnected union of two intervals. The proof is described in heuristic terms in $\$ 2$ following the statement of Theorem 1.

Received by the editors November 14, 1983.

1980 Mathematics Subject Classification. Primary 34C35; Secondary 35K55, 92A05.

Key words and phrases. Traveling wave solution, diffusive Lotka-Volterra system, heteroclinic orbit, shooting argument. 
Many investigators have used system (1) and related equations for ecological modeling and simulations in an effort to understand the most basic features of a spatially distributed interaction. For a survey see [21, Chapter $10 ; 20, \S \S 4.1,4.11$; or 9, Chapter 5]. A number of factors motivate the search for the existence of traveling wave solutions. The idea that a reaction-diffusion equation can give rise to a moving zone of transition between equilibrium states, i.e. a traveling wave front, goes back to Fisher [10] and Kolmogorov, Petrovsky and Piscounov [17]. They found a traveling wave solution to a reaction diffusion equation from population genetics which models the advance of an advantageous gene. By taking $w \equiv 0$ in system (1), the system reduces to the equation studied by Fisher. Thus, it is interesting to see if a similar kind of solution can be found in system (1).

Dubois [5] used a system like (1) without the logistic growth term to model spatial structure of plankton populations within a favorable habitat whose size far exceeds the plankton patch size. Dubois selected parameter values to fit data for oceanic plankton. In simulations he found wave forms which advance across the habitat. This suggests that traveling wave solutions are important in models of oceanic plankton distribution. An unusual feature of the traveling waves found in this paper is that the waves may have damped oscillations on the "after" side of the transition zone. The precise statement may be found in Theorem 1. After the transition zone of these traveling wave solutions has passed, an observer would measure successively higher and lower densities of the species. These regions of successively higher and lower densities, corresponding to the maxima and minima of the damped oscillation, would move with the speed $c$ of the traveling wave. This is qualitatively similar to the observations of Wyatt [24] of plankton patches moving through the Southern Bight.

In [1] Chow and Tam have investigated the possibility of traveling wave solutions to a Lotka-Volterra reaction-diffusion system with $D_{1}=0$. Using a formal analysis, they showed that for a special initial condition for the Cauchy problem corresponding to (1), and for a specific choice of $c$, solutions to (1) asymptotically possess some of the characteristics of a traveling wave solution. However, they were not able to do the analysis for $D \neq 0$. In [6] the author has obtained a rigorous proof for the existence of traveling wave solutions when $D=0$. The shooting technique employed here is motivated by the proof in [6].

Analysis of the space-independent Lotka-Volterra model provides a motivation which leads directly to the statement of Theorem 1. Before discussing this it will be helpful to express the equations in dimensionless variables and parameters. Taking $U=u / K, W=(B / A) w, t^{\prime}=A t, x^{\prime}=x / \sqrt{D_{2} / A}, D=D_{1} / D_{2}, \alpha=E K / A, \beta=$ $C / E K$; and dropping the primes on $x, t$ for convenience, we obtain

$$
U_{t}=D U_{x x}+U(1-U-W), \quad W_{t}=W_{x x}+\alpha W(U-\beta) .
$$

All constants are positive. For a system in which the predator can survive and establish a nonzero population level, the death rate of the predator, $C$, should be less than the growth rate $E K$ of the predator at the maximum sustainable prey population $K$. Thus, $0<\beta<1$. The reaction term of system (2) has three equilibrium points. The equilibria $(0,0)$, representing the absence of both species, and $(1,0)$, 
representing the population of the prey at the environmental carrying capacity in the absence of predators, are both unstable. The equilibrium $(\beta, 1-\beta)$, representing the time constant coexistence of both species, is stable. If $4 \alpha<\beta /(1-\beta)$, then $(\beta, 1-\beta)$ is a stable node; while if $4 \alpha>\beta /(1-\beta)$, then $(\beta, 1-\beta)$ is a stable spiral point. Using the well-known, e.g. in [16, p. 262], Liapunov function

$$
V(u, w)=\alpha[u-\beta-\beta \log (u / \beta)]+[w-(1-\beta)-(1-\beta) \log (w /(1-\beta))],
$$

it is easily seen that $(\beta, 1-\beta)$ is globally asymptotically stable in the positive quadrant. Thus, solutions of

$$
d U / d t=U(1-U-W), \quad d W / d t=\alpha W(U-\beta)
$$

initially near the unstable equilibrium $(1,0)$ approach the coexistence equilibrium $(\beta, 1-\beta)$. In ecological terms this means that if the prey is near its environmental carrying capacity, the introduction of even a small number of predators will drive the system to a new stable state of coexistence with a reduced number of prey.

If the species are free to move about in a 1-, 2-, or 3-dimensional habitat, these transitions from one equilibrium state to another may take place in a spatially mediated way. For mathematical and conceptual simplicity, we consider a 1-dimensional habitat. For instance, if the linear habitat, e.g. a coastline, river, or channel, is initially uniformly saturated with prey at the carrying capacity of the environment, the introduction of a few predators at one end of the habitat may result in a "wave of invasion" of predators. That is, there could be a zone of transition from the $(1,0)$ state to a decreased level of prey $\beta$ and increased level of predators $1-\beta$. If this transition zone moves across the habitat, we call it a traveling wave front.

Systems of reaction-diffusion equations from neurophysiology, chemistry, and epidemiology possess traveling wave solutions; for a survey see [9]. More recently, traveling wave solutions have been established for systems from neurophysiology [22] and mathematical ecology [3, 12, 13]. In [13] Gardner has shown the existence of traveling wave solutions in a predator-prey system different from the one considered here. In [13] the traveling wave solutions are a transition between two stable equilibria, one representing absence of both species and the other equilibrium representing a stable coexistence. The proof uses the Conley index.

The traveling wave solutions found in this paper differ from the fronts found in most of the other work cited above in that they are not necessarily monotone functions. In [22] Pauwelussen has found nonmonotone traveling wave front solutions in a model from neurophysiology. In [13] the traveling wave front solutions may also be nonmonotone. In both cases the method of proof is different from that employed here.

The organization of the paper is as follows: In $\$ 2$ we state Theorem 1, which is our main result, a Proposition (a variant of Wazewski's Theorem), which is the main tool used in the proof, and a heuristic description of the proof. The ideas are topologically quite simple, but the geometry of $\mathbf{R}^{4}$ makes their presentation complicated. Although the techniques used here are motivated by the proof in [6], the present paper is self-contained and independent of that work. 
2. Main results and description of the proof. The main goal of this paper is to establish the existence of traveling wave front solutions of system (2). These are solutions having the special form

$$
U(t, x)=u(x+c t), \quad W(t, x)=w(x+c t) .
$$

The functions $u, w$ are functions of a single variable $s=x+c t$ and the wave speed parameter $c$ is positive. Then (2) becomes

$$
c u^{\prime}=D u^{\prime \prime}+u(1-u-w), \quad c w^{\prime}=w^{\prime \prime}+\alpha w(u-\beta) .
$$

Here' denotes differentiation with respect to the wave variable $s$. Recalling the ecological motivation, we require the traveling wave solutions to be nonnegative. We also require that the solutions satisfy the boundary conditions:

$$
u(-\infty)=1, \quad u(+\infty)=\beta, \quad w(-\infty)=0, \quad w(+\infty)=1-\beta,
$$

where $u(-\infty)=\lim _{s \rightarrow-\infty} u(s)$, etc. We write (3) as a first order system in $\mathbf{R}^{4}$ :

$$
\begin{array}{rlrl}
u^{\prime} & =v, & v^{\prime} & =(c / D) v-(1 / D) u(1-u-w), \\
w^{\prime} & =z, & z^{\prime}=c z-\alpha w(u-\beta) .
\end{array}
$$

We then have

THEOREM 1. (a) If $c>c^{*}=\sqrt{4 \alpha(1-\beta)}$ and $D \leqslant 1$, then nonnegative solutions of (5) satisfying boundary conditions (4) exist.

(b) If $c<\sqrt{4 \alpha(1-\beta)}$, then nonnegative solutions of system (5) satisfying boundary conditions (4) do not exist.

There is a value $\alpha^{*}=\alpha^{*}(\beta, c, D)$ such that if traveling wave front solutions exist and:

(1) $\alpha \leqslant \alpha^{*}$, the functions $u, w$ approach $\beta, 1-\beta$ monotonely for large $s$;

(2) $\alpha>\alpha^{*}$, the functions $u, w$ have exponentially damped oscillations about $(\beta, 1-\beta)$ for large $s$.

Also we have the estimate $\alpha^{*}>\beta / 4(1-\beta)$.

The principal tools necessary for the existence proof in this paper are Wazewski's Theorem, the Stable Manifold Theorem, and LaSalle's Invariance Principle. A proof of the Stable Manifold Theorem is in [14, Theorems 6.1, 6.2, pp. 242, 243]. A proof of the Invariance Principle is in [18].

In this section a variant of Wazewski's Theorem, which is a formalization and extension of the "shooting method", is stated. This Proposition recognizes that the flow defined by the solutions of a differential equations gives a topological mapping between regions of phase space. The statement and proof of Wazewski's Theorem are variants of those given in [2]. The notation is the same as in [2].

Consider the differential equation

$$
\mathbf{y}^{\prime}=\mathbf{f}(\mathbf{y}), \quad \mathbf{y} \in \mathbf{R}^{n}\left({ }^{\prime}=d / d s\right)
$$

where $\mathbf{f}: \mathbf{R}^{n} \rightarrow \mathbf{R}^{n}$ is continuous and satisfies a Lipschitz condition. Let $\mathbf{y}\left(s ; \mathbf{y}_{0}\right)$ be the unique solution of (5) satisfying $\mathbf{y}\left(0 ; \mathbf{y}_{0}\right)=\mathbf{y}_{0}$. For convenience set $\mathbf{y}\left(s, \mathbf{y}_{0}\right)=\mathbf{y}_{0} \cdot s$. Let $U \cdot S$ be the set of points $\mathrm{y}_{0} \cdot s$ where $\mathrm{y}_{0} \in U, s \in S$. 

let

In order to state the proposition, some definitions are necessary. Given $W \subseteq \mathbf{R}^{n}$,

$$
W^{-}=\left\{\mathbf{y}_{0} \in W \mid \text { any } s>0, \mathbf{y}_{0} \cdot[0, s) \nsubseteq W\right\} .
$$

$W^{-}$is called the immediate exit set of $W$. Given $\Sigma \subseteq W$, let

$$
\Sigma^{0}=\left\{\mathbf{y}_{0} \in \Sigma \mid \text { there is an } s_{0}=s_{0}\left(\mathbf{y}_{0}\right) \text { such that } \mathbf{y}_{0} \cdot s_{0} \notin W\right\} \text {. }
$$

For $\mathbf{y}_{0} \in \Sigma^{0}$ define

$$
T\left(\mathbf{y}_{0}\right)=\sup \left\{s \mid \mathbf{y}_{0} \cdot[0, s] \subseteq W\right\} .
$$

$T\left(y_{0}\right)$ is called an exit time. Note that $y_{0} \cdot T\left(y_{0}\right) \in W^{-}$. Note also that $T\left(y_{0}\right)=0$ if and only if $y_{0} \in W^{-}$. The notation $\operatorname{cl}(W)$ is used for the closure of $W$.

Proposition 2. Suppose:

(1) If $\mathbf{y}_{0} \in \Sigma$ and $\mathbf{y}_{0} \cdot[0, s] \subseteq \operatorname{cl}(W)$, then $\mathbf{y}_{0} \cdot[0, s] \subseteq W$.

(2) If $\mathrm{y}_{0} \in \Sigma, \mathrm{y}_{0} \cdot s \in W, \mathrm{y}_{0} \cdot s \notin W^{-}$, then there is an open set $V_{s}$ about $\mathrm{y}_{0} \cdot s$ disjoint from $W^{-}$.

(3) $\Sigma=\Sigma^{0}, \Sigma$ is compact, and $\Sigma$ intersects a trajectory of (6) only once.

Then the mapping $F\left(\mathrm{y}_{0}\right)=\mathrm{y}_{0} \cdot T\left(\mathrm{y}_{0}\right)$ is a homeomorphism from $\Sigma$ to its image on $W^{-}$.

The proof is given in $\$ 3 \mathrm{~A}$.

The solutions of interest of system (5) have limiting values as $s$ tends to plus or minus infinity. This means that the solutions of interest correspond to orbits in phase space connecting one critical point to another. For the boundary values (4), the task is to find a trajectory of (5) connecting $(1,0,0,0)$ to $(\beta, 0,1-\beta, 0)$ in $\mathbf{R}^{4}$, with $u, w>0$. In $\S 3 C$ we show that the unstable manifold at $(1,0,0,0)$ is three dimensional. In $\S 3 \mathrm{~F}$ we show that the stable manifold at $(\beta, 0,1-\beta, 0)$ is two dimensional. Roughly speaking, the proof identifies the connection between the critical points as the intersection of those manifolds in 4-dimensional phase space. The "transversality" of such an intersection in general "explains" the existence of the connection for a whole half line of parameter values. Indeed, the "transversality" of such an intersection shows that the connection exists for parameter values outside the range specified in the Theorem. Nevertheless, these connections are meaningless in the biological context because the connecting orbit enters the region $w<0$, as shown in §3B. The proofs in this paper are intended to capture the existence of biologically meaningful connections only.

We use Proposition 2 to "capture" the intersection in the following way. We take $W$ to be a hypercone in 4-dimensional phase space containing the 2-dimensional stable manifold and excluding the complementary 2-dimensional unstable manifold. The vertex of the hypercone is at $(\beta, 0,1-\beta, 0)$. This is pictured schematically in Figure 1 by considering an analogous situation in 3-dimensional space. The set $W$ is described precisely in §3B.

The set $W^{-}$is the lateral surface of the hypercone excluding the vertex. Notice that in Figure 1 this is not simply connected. We show in Appendix 2 that in the situation given precisely in $\S 3$, the set $W^{-}$is not simply connected. 


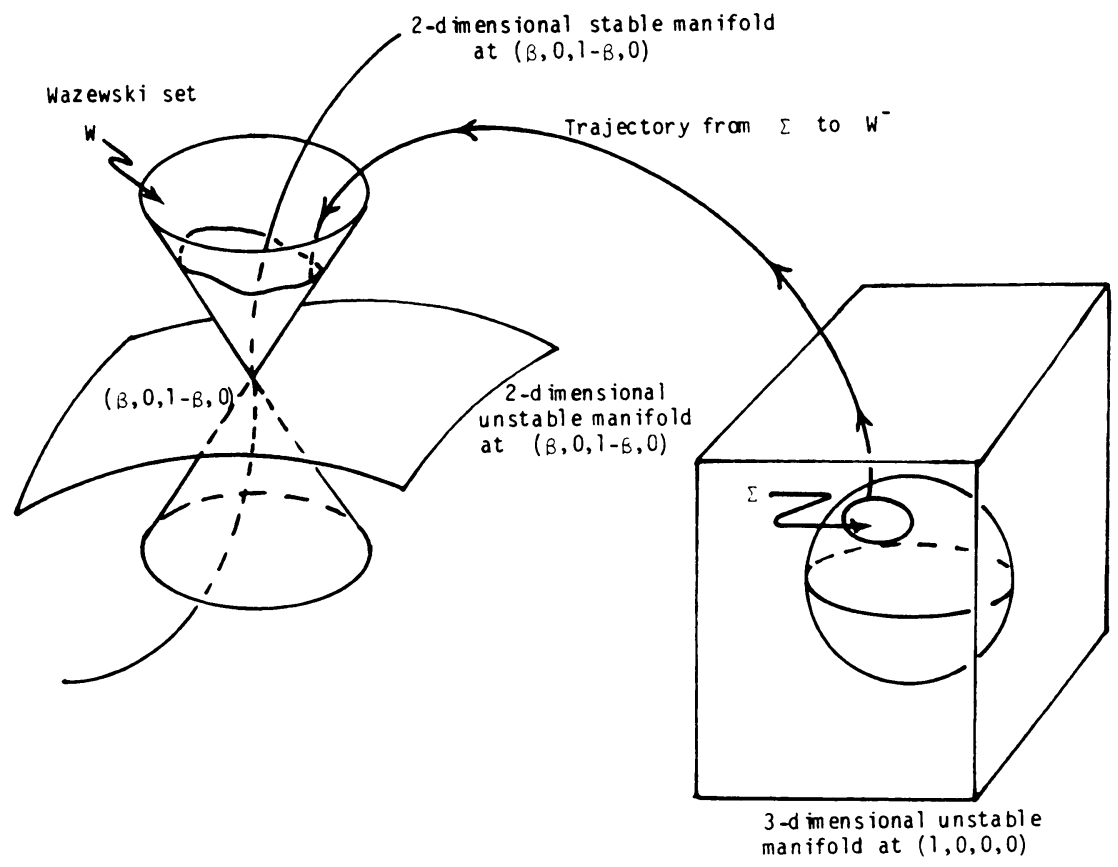

Figure 1.

Schematic diagram of the proof of existence of the heteroclinic connection.

The unstable manifold of $(5)$ at $(1,0,0,0)$ is 3 -dimensional. We take a small sphere surrounding $(1,0,0,0)$ in this manifold. In $\S 3 \mathrm{D}$ we identify a simply connected patch $\Sigma$ on this sphere and show that the boundary of this patch is mapped by the flow of (5) to a loop surrounding $(\beta, 0,1-\beta, 0)$ in $W^{-}$. See Figure 1 for a schematic picture of this.

Thus, by Proposition 2, if every trajectory through the patch $\Sigma$ left $W$ by the exit set $W^{-}$, we would have a simply connected patch $\Sigma$ mapped homeomorphically to a non-simply-connected image on $W^{-}$. This contradiction shows that there must be a trajectory through $\Sigma$ which does not leave $W$. After showing in $\S 3 \mathrm{E}$ that this trajectory is bounded and satisfies $u, w>0$, we use a Liapunov function to show that the trajectory approaches $(\beta, 0,1-\beta, 0)$. This finishes the proof.

This is a typical sort of shooting argument, although it is a "simple connectedness" one. Previous shooting arguments to establish the existence of traveling wave solutions in reaction-diffusion equations, e.g. in $[8,15,23]$, have been "disconnection" arguments. That is, the unstable manifold at the target point is 1-dimensional, while the stable manifold is $(n-1)$-dimensional. This provides a "splitting" of space near the target point and a disconnection type shooting argument is possible. In [19] McLeod and Serrin have used a topological argument equivalent to simple connectedness to show the existence of a pair of parameter values which allow the solution of a boundary value problem. In the present situation the target point has a 2-dimensional stable manifold and a 2-dimensional unstable manifold. This requires an argument involving a higher connection type, namely simple connectedness. The 
possibility of a spiral on the stable manifold at $(\beta, 0,1-\beta, 0)$ prevents us from "turning the shooting around" to use a disconnection argument at the target point $(1,0,0,0)$.

3. Proof of Theorem. For convenience we have divided the proof into several subsections $(\mathrm{A}-\mathrm{F})$. In $\mathrm{A}$ we have the proof of Proposition 2 . In $\mathrm{B}$ we define the Wazewski set $W$ and examine the exit set $W^{-}$. Some tedious details are deferred to Appendix 1. In $C$ we examine the linearization of (5) about $(1,0,0,0)$. We also present some lemmas which capture the behavior of trajectories on the strongly unstable manifold at $(1,0,0,0)$. In $\mathrm{D}$ we present some simple lemmas which allow us to construct the set $\Sigma$. In $\mathrm{E}$ we finish the proof of existence of the waves, including some topological arguments which are the main part of the proof. Again, some technical details are deferred to Appendix 2. In F we examine the stable manifold of the system $(5)$ at $(\beta, 0,1-\beta, 0)$ which establishes the asymptotic behavior of the waves as $s \rightarrow \infty$.

A. In this section a variant of Wazewski's Theorem, which is a formalization and extension of the "shooting method", is proved. This Proposition recognizes that the flow defined by the solutions of a differential equation gives a topological mapping between regions of phase space. The statement and proof of Wazewski's Theorem are modifications of those in [2, Theorem 2.3, p. 24].

Consider the differential equation

$$
\mathbf{y}^{\prime}=\mathbf{f}(\mathbf{y}), \quad \mathbf{y} \in \mathbf{R}^{n}\left({ }^{\prime}=d / d s\right),
$$

where f: $\mathbf{R}^{n} \rightarrow \mathbf{R}^{n}$ is continuous and satisfies a Lipschitz condition. Recall the definitions of $W^{-}, \Sigma^{0}$, etc. given in $\$ 2$. For convenience we restate Proposition 2.

Proposition 2. Suppose:

(1) If $\mathbf{y}_{0} \in \Sigma$, and $\mathbf{y}_{0} \cdot[0, s] \subseteq \mathrm{cl}(W)$, then $\mathbf{y}_{0} \cdot[0, s] \subseteq W$.

(2) If $\mathrm{y}_{0} \in \Sigma, \mathrm{y}_{0} \cdot s \in W, \mathrm{y}_{0} \cdot s \notin W^{-}$, then there is an open set $V_{s}$ about $\mathrm{y}_{0} \cdot s$ disjoint from $W^{-}$.

(3) $\Sigma=\Sigma^{0}, \Sigma$ is compact, and $\Sigma$ intersects a trajectory of (6) only once.

Then the mapping $F\left(\mathrm{y}_{0}\right)=\mathrm{y}_{0} \cdot T\left(\mathrm{y}_{0}\right)$ is a homeomorphism from $\Sigma$ to its image on $W^{-}$.

A set $W \subseteq \mathbf{R}^{n}$ satisfying (1) and (2) is called a Wazewski set.

Proof. $F$ is one-to-one because $\Sigma$ intersects a trajectory of (6) only once. The mapping of $\Sigma$ to its image on $W^{-}$is, by definition, an onto mapping. If we show that $T\left(\mathrm{y}_{0}\right)$ is continuous, then the composition $\mathrm{y}_{0} \cdot T\left(\mathrm{y}_{0}\right)=F\left(\mathrm{y}_{0}\right)$ is a continuous, one-toone mapping from a compact space to a Hausdorff space; that is, $F$ is a homeomorphism.

Hypothesis (1) guarantees that $T\left(y_{0}\right)$ is upper semicontinuous. Let $\varepsilon>0$. Then $\mathbf{y}_{0} \cdot\left[T\left(\mathbf{y}_{0}\right), T\left(y_{0}\right)+\varepsilon\right] \nsubseteq W$. Using hypothesis (1), there is an $s^{\prime} \in\left[T\left(y_{0}\right), T\left(y_{0}\right)+\varepsilon\right]$ such that $\mathrm{y}_{0} \cdot s^{\prime} \notin \operatorname{cl}(W)$. Let $V$ be a neighborhood of $y_{0} \cdot s^{\prime} \operatorname{disjoint}$ from $\operatorname{cl}(W)$. Let $U$ be a neighborhood of $\mathbf{y}_{0}$ such that $U \cdot s^{\prime} \subseteq V$. For $\mathbf{y}_{1} \in \Sigma \cap U, \mathbf{y}_{1} \cdot s^{\prime} \in V$. Therefore $T\left(\mathrm{y}_{1}\right) \leqslant s^{\prime} \leqslant T\left(\mathrm{y}_{0}\right)+\varepsilon$. 
Hypothesis (2) guarantees that $T\left(\mathrm{y}_{0}\right)$ is lower semicontinuous. Let $\mathrm{y}_{0} \in \Sigma^{0}, \varepsilon>0$. Then $\mathrm{y}_{0} \cdot\left[0, T\left(\mathrm{y}_{0}\right)-\varepsilon\right] \subseteq W$. By hypothesis (2), for each $s \in\left[0, T\left(\mathrm{y}_{0}\right)-\varepsilon\right]$ there is a neighborhood $V_{s}$ of $\mathrm{y}_{0} \cdot s$ disjoint from $W^{-}$. Since the set $\mathrm{y}_{0} \cdot\left[0, T\left(\mathrm{y}_{0}\right)-\varepsilon\right]$ is compact, a finite number of $V_{s}$ cover it. Let $V$ be the union of this finite collection. Let $U$ be a neighborhood of $\mathbf{y}_{0}$ such that $U \cdot\left[0, T\left(\mathbf{y}_{0}\right)-\varepsilon\right] \subseteq V$. If $\mathbf{y}_{0} \in U$, then $\mathbf{y}_{1} \cdot\left[0, T\left(\mathbf{y}_{0}\right)-\varepsilon\right] \cap W^{-}=\varnothing$. Therefore, $T\left(\mathbf{y}_{1}\right)>T\left(\mathbf{y}_{0}\right)-\varepsilon$.

B. We first note that if $(u(s), v(s))$ is a solution of the system

$$
u^{\prime}=v, \quad v^{\prime}=c / D-(1 / D) u(1-u),
$$

then $(u(s), v(s), 0,0)$ is a solution of system (5). That is, the set $\mathscr{V}=\{(u, v, w, z) \mid w$ $=0, z=0\}$ is an invariant manifold in the phase space of (5). Likewise if $(w(s), z(s))$ is a solution of

$$
w^{\prime}=z, \quad z^{\prime}=c z+\alpha \beta w,
$$

then $(0,0, w(s), z(s))$ is a solution of (5). Thus, the set $\mathscr{W}=\{(u, v, w, z) \mid u=v=0\}$ is an invariant manifold in the phase space of (5).

The choice of the Wazewski set in [6] motivates the choice of the Wazewski set $W$ defined here. It will be the complement of four blocks in $\mathbf{R}^{4}$, two of which are chosen so that $z^{\prime}$ has the same sign as $z$ so solutions entering these blocks would not have $z \rightarrow 0$ as $s \rightarrow \infty$. The other pair of blocks are chosen so that $v^{\prime}$ has the same sign as $v$ and so solutions entering these blocks will not have $v \rightarrow 0$ as $s \rightarrow \infty$.

We define

$$
\begin{aligned}
P & =\{(u, v, w, z) \mid u<\beta, w>1-\beta, z>0\}, \\
Q & =\{(u, v, w, z) \mid u>\beta, w<1-\beta, z<0\}, \\
R & =\{(u, v, w, z) \mid u<\beta, 1-u-w>0, v<0\}, \\
S & =\{(u, v, w, z) \mid u>\beta, 1-u-w<0, v>0\}, \\
W & =\mathbf{R}^{4} \backslash(P \cup Q \cup R \cup S) .
\end{aligned}
$$

Note that $P \cap R \neq \varnothing, Q \cap S \neq \varnothing$, while all other pairwise intersections are empty.

A consequence of the definition of the immediate exit set $W^{-}$is that $W^{-}$is contained in the boundary of $W$. The description of the boundary of $W$ is in terms of the boundary of its complement ( $P \cup Q \cup R \cup S$ ). This description is complicated by the fact that the pairs of blocks $P, R$, and $Q, S$ overlap. This means $\partial P \cap R \neq \varnothing, \partial R \cap P \neq \varnothing, \partial Q \cap S \neq \varnothing$, and $\partial S \cap Q \neq \varnothing$. Then

$$
\partial W=(\partial P \backslash R) \cup(\partial Q \backslash S) \cup(\partial R \backslash P) \cup(\partial S \backslash Q)
$$

and

$$
W^{-}=\partial W \backslash\left(\{(\beta, 0,1-\beta, 0)\} \cup J_{1} \cup J_{2}\right),
$$

where

$$
\begin{aligned}
J_{1}= & \{(u, v, w, z) \mid u=\beta, v>0, w \leqslant 0, z=0\} \\
& \cup\{(u, v, w, z) \mid u>\beta, w \leqslant 0,1-u-w>0, z=0\} \\
& \cup\{(u, v, w, z) \mid u>\beta, w<0,1-u-w \leqslant 0, v<0, z=0\} \\
& \cup\{(u, v, w, z) \mid u=1, v \leqslant 0, w=0, z=0\}
\end{aligned}
$$


and

$$
\begin{aligned}
J_{2}= & \{(u, v, w, z) \mid u<0, w>1-\beta, 1-u-w \geqslant 0, z<0, v=0\} \\
& \cup\{(u, v, w, z) \mid u<0,1-u-w>0, w \leqslant 1-\beta z<0, v=0\} \\
& \cup\{(u, v, w, z) \mid u \leqslant 0,1-u-w>0, w<1-\beta, v=0\} \\
& \cup\{(u, v, w, z) \mid u=0, v=0, w \geqslant 1-\beta, z<0\} .
\end{aligned}
$$

Although this appears formidable, the explanation is simple. $J_{1}$ is the set of points on $\partial W$ which do not exit from $W$ into $Q, R$, or $S$. This can happen two ways. Some points in the invariant manifold $\mathscr{V}$ may not enter $R$ or $S$ immediately. Of course, they will remain in $\mathscr{V}$ for all time and so do not enter $Q$. Points on $\partial W$ with $z=0$, $w<0$ will enter $W$ from $Q$ and so will not be immediate exit points.

$J_{2}$ is the set of points on $W$ which do not exit immediately from $W$ into $P$ or $R$. This can also happen in two ways. Some points on the invariant manifold $\mathscr{W}$ do not immediately enter $P$, but remain in $\mathscr{W}$ for all time, so do not enter $R$. Points on $\partial W$ with $v=0,1-u-w>0$ and $u<0$ will enter $W$ from $R$ and so will not be immediate exit points. Details of the proof that $W^{-}$is the set described above are tedious. The proof is deferred to Appendix 1 . There we only examine the portion $\partial R \backslash P$ of $\partial W$ as an example. This will show why $J_{2}$ must be excluded from $\partial W$ to obtain $W^{-}$. The complete proof may be found in [7].

$W^{-}$is not a simply connected set. It has a "hole" which is the removed point $(\beta, 0,1-\beta, 0)$. This is not geometrically obvious and is proved in Appendix 2.

C. The eigenvalues of the linearization of $(3)$ at $(1,0,0,0)$ are

$$
\begin{array}{ll}
\lambda_{1}=\left(c-\sqrt{c^{2}+4 D}\right) / 2 D, & \lambda_{2}=\left(c-\sqrt{c^{2}-4 \alpha(1-\beta)}\right) / 2, \\
\lambda_{3}=\left(c+\sqrt{c^{2}-4 \alpha(1-\beta)}\right) / 2, & \lambda_{4}=\left(c+\sqrt{c^{2}+4 D}\right) / 2 D .
\end{array}
$$

If $c^{2}<4 \alpha(1-\beta)$, then $\lambda_{2}, \lambda_{3}$ are a complex conjugate pair of eigenvalues with positive real part. The eigenvalues $\lambda_{1}, \lambda_{4}$ are always real and $\lambda_{1}<0<\lambda_{4}$. If $D \leqslant 1$ then $\lambda_{4} \geqslant \operatorname{Re}\left(\lambda_{2}\right), \operatorname{Re}\left(\lambda_{3}\right)$. Using [14, Theorems 6.1, 6.2, p. 242] a single trajectory of system (3) approaches $(1,0,0,0)$ tangent to an eigenvector $\mathbf{x}_{4}$ corresponding to the eigenvalue $\lambda_{4}$ as $s \rightarrow-\infty$. An easy calculation shows $x_{4}=\left(-1,-\lambda_{4}, 0,0\right)$.

As previously mentioned, if $(u(s), v(s))$ is a solution to the system

$$
u^{\prime}=v, \quad v^{\prime}=(c / D) u-(1 / D) u(1-u)
$$

then $(u(s), v(s), 0,0)$ is a solution of system (5). Equation (7) is the system whose solutions yield traveling wave solutions to the parabolic partial differential equation

$$
U_{t}=D U_{x x}+U(1-U),
$$

sometimes called Fisher's equation.

The eigenvalues of the linearization of (7) at $(1,0)$ are the $\lambda_{1}, \lambda_{4}$ defined previously. An eigenvector associated with $\lambda_{4}$ for (7) is $\left(-1,-\lambda_{4}\right)$. There is then a single trajectory of solutions of $(7)$ which approach $(1,0)$ tangent to $\left(-1,-\lambda_{4}\right)$ as $s \rightarrow-\infty$. 
This implies that the single trajectory of solutions of (5) approaching $(1,0,0,0)$ as $s \rightarrow-\infty$ is actually contained in the invariant manifold $\mathscr{V}=\{(u, v, w, z) \mid w=z=$ $0\}$. Then a solution corresponding to this trajectory cannot approach $(\beta, 0,1-\beta, 0)$ as $s \rightarrow+\infty$, so it is not the desired traveling wave front solution of (5). Recalling that if $c^{2}<4 \alpha(1-\beta)$ we have two complex eigenvalues with positive real part, any other solution of (5) approaching $(1,0,0,0)$ as $s \rightarrow-\infty$ must spiral in toward $(1,0,0,0)$. This spiraling of solutions cannot take place with $w>0$. If it did then $u(s)$ would have some local maximum value greater than 1 . At this local maximum $u^{\prime \prime}(s)<0, u^{\prime}(s)>1$, and $w(s) \geqslant 0$. Then

$$
0=D u^{\prime \prime}-c u^{\prime}(s)+u(s)(1-u(s)-w(s))<0,
$$

which is a contradiction. Thus a trajectory spiraling in toward $(1,0,0,0)$ as $s \rightarrow-\infty$ must have $w(s) \leqslant 0$ for some $s$. This violates the requirement that traveling wave front solutions be nonnegative. This shows that if $c^{2}<4 \alpha(1-\beta)$, traveling wave front solutions do not exist.

We now require $c^{2}>4 \alpha(1-\beta)$. With this restriction there are four distinct real eigenvalues, which may be written in increasing order: $\lambda_{1}<0<\lambda_{2}<\lambda_{3}<\lambda_{4}$. Eigenvectors $\mathbf{x}_{2}, \mathbf{x}_{3}, \mathbf{x}_{4}$ associated with $\lambda_{2}, \lambda_{3}, \lambda_{4}$, respectively, are

$$
\left(\begin{array}{c}
-1 \\
-\lambda_{2} \\
-p\left(\lambda_{2}\right) \\
-\lambda_{2} p\left(\lambda_{2}\right)
\end{array}\right), \quad\left(\begin{array}{c}
-1 \\
-\lambda_{3} \\
-p\left(\lambda_{3}\right) \\
-\lambda_{3} p\left(\lambda_{3}\right)
\end{array}\right), \quad\left(\begin{array}{c}
-1 \\
-\lambda_{4} \\
0 \\
0
\end{array}\right),
$$

where $p(\lambda)=(D-1) \lambda^{2}-(1+\alpha(1-\beta))$.

Using [14, Theorems $6.1,6.2$, p. 242] we can say there is a strongest unstable manifold $\mathscr{U}$ tangent to $\mathbf{x}_{4}$ at $(1,0,0,0)$. There is also a 2-dimensional strongly unstable manifold $\mathscr{U}_{2}$ tangent to the span of $\mathbf{x}_{4}$ and $\mathbf{x}_{3}$ at $(1,0,0,0)$. Finally there is a 3-dimensional unstable manifold $\mathscr{U}_{3}$ tangent to the span of $x_{4}, x_{3}$ and $x_{2}$ at $(1,0,0,0)$.

We now define $\Sigma$ as a quadrilateral on a sphere surrounding $(1,0,0,0)$ in the 3-dimensional unstable manifold $\mathscr{U}_{3}$. The definition of $\Sigma$ will show that the hypotheses of Proposition 2 are satisfied. In addition, we obtain information about the image of the boundary of $\Sigma$ under the mapping $F\left(y_{0}\right)=y_{0} \cdot T\left(y_{0}\right)$ defined in $\S 2$.

We define the quadrilateral by specifying four sides in order. The first side is an arc of the circle which is the intersection of the 2-dimensional strongly unstable manifold $\mathscr{U}_{2}$ with the small sphere. The next two sides are arcs of the circles which are the intersections of the sphere with the hyperplanes $v=0$ and $z=0$. The last side will be obtained by a continuity argument. We now prove a series of lemmas which will establish the vertices of this quadrilateral and thus define the arcs.

Throughout the remainder of the paper we use the notation $\mathbf{y}\left(s ; \mathbf{y}_{0}\right)$ for a solution of system (5) satisfying the initial condition $\mathbf{y}\left(0 ; \mathbf{y}_{0}\right)=\mathbf{y}_{0} . \mathbf{y}_{0}$ is a vector $\left(u_{0}, v_{0}, w_{0}, z_{0}\right)$. We use the notation $u\left(s, \mathbf{y}_{0}\right)$ for the first coordinate function of $\mathbf{y}\left(s ; \mathbf{y}_{0}\right)$, and similarly for the other three coordinate functions. When the initial condition is clear from the context, we write $y(s)$, etc. 
Lemma 3. Consider a solution $\mathrm{y}\left(s, \mathrm{y}_{0}\right)$ with $\mathrm{y}_{0} \in \mathscr{U}_{1}$ and $u_{0}<1$. Then there is $a$ finite $s_{0}$ such that $u\left(s_{0}, y_{0}\right)<\beta, v\left(s_{0}, y_{0}\right)<0$. That is, the solution enters the region $R$.

Proof. Recall that earlier in the section we showed that the strongest unstable manifold $\mathscr{U}_{1}$ is contained in the invariant manifold $\mathscr{V}$. The solutions of (5) in $\mathscr{V}$ are given by $(u(s), v(s), 0,0)$, where $(u(s), v(s))$ is a solution of (7). To analyze trajectories on $\mathscr{U}_{1}$, then, it suffices to use the phase plane diagram for (7).

The vector field of (7) shows that a solution of (7) which approaches $(1,0)$ as $s \rightarrow-\infty$ (that is, a trajectory on $\mathscr{U}_{1}$ ), and which has a point such that $u_{0}<1$, must approach $(1,0)$ tangent to the eigenvector $\left(-1,-\lambda_{4}\right)$ in the region $u<1, v<0$. Choose $m, n$ so that $0<n<c / D<\lambda_{4}<m$. Since (7) is autonomous, we may rescale the time and choose a new initial condition $y_{0}$ if necessary so that

$$
m(u(0)-1)<v(0)<n(u(0)-1) .
$$

It is easy to check that, with the choice of $m, n$ made above, the vector field of (7) points inward to the region $m(u-1)<v<n(u-1)$ in the strip $0<u<1, v<0$. See Figure 2. Therefore, in the region $0<u<1, v<0$, the trajectory of a solution starting on $\mathscr{U}_{1}$ satisfies

$$
m(u(s)-1)<v(s)<n(u(s)-1) .
$$

Since $u^{\prime}=v$, integrate to obtain

$$
1-C_{1} e^{m s}<u(s)<1-C_{2} e^{n s}
$$

for $s$ such that $u(s)$ satisfies $0<u<1$. Then for large enough $s_{0}, u\left(s_{0}\right)<\beta$, $v\left(s_{0}\right)<0$. Q.E.D.

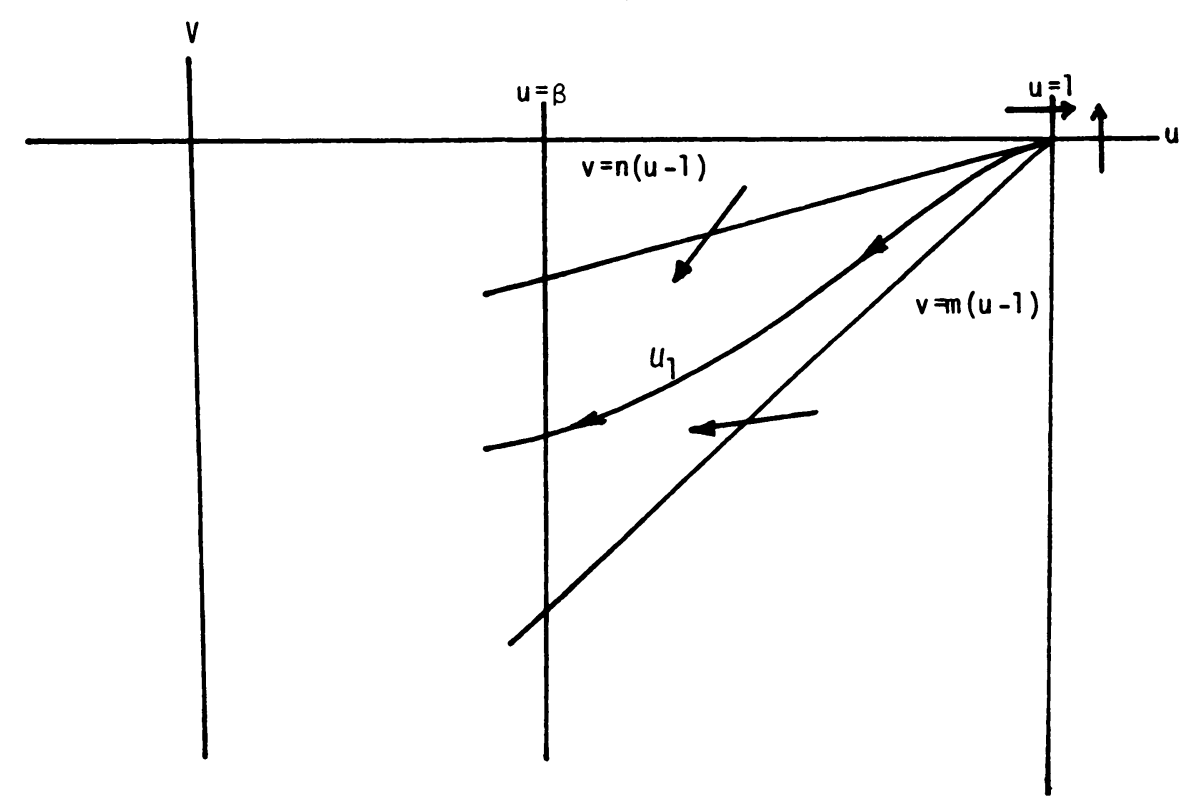

FIgURE 2. Phase plane diagram for Lemmas 3 and 4. 
LEMMA 4. A solution $\mathrm{y}\left(s, \mathrm{y}_{0}\right)$ on $\mathscr{U}_{1}$ which approaches $(1,0,0,0)$ as $s \rightarrow-\infty$ in the region $u>1, v>0$ will remain in that region for all $s$.

Proof. The phase plane diagram in Figure 2 shows that the region $u>1, v>0$ is positively invariant for the flow of (7). Also, trajectories of (7) which approach $(1,0)$ as $s \rightarrow-\infty$ in the region $u>1, v>0$ do so tangent to the eigenvector $\left(1, \lambda_{4}\right)$. This establishes the lemma.

We next analyze trajectories of solutions on the strongly unstable manifold $\mathscr{U}_{2}$.

LEMMA 5. Any trajectory which has a point (corresponding to $s=0$ without loss of generality) such that $w(0)>0, z(0)>(c / 2) w(0)$ will have $w(s)>0$ and $z(s)>$ $(c / 2) w(s)$ for all $s>0$ such that $u(s) \leqslant 1$.

In particular, this is true for trajectories on $\mathscr{U}_{2}$ approaching $(1,0,0,0)$ tangent to the vector $\mathbf{x}_{3}$ in the region where $u<1$.

Proof. Suppose there was some $s$ such that $u(s)<1, z(s)=(c / 2) w(s)$. Let

$$
s_{1}=\inf \{s>0 \mid z(s)=(c / 2) w(s), u(s)<1\} .
$$

Because $w(0)>0$ and $w^{\prime}(s)=z(s)>(c / 2) w(s)$ for $s \in\left[0, s_{1}\right]$, we have $w\left(s_{1}\right)>0$. At $s=s_{1}$,

$$
z^{\prime}\left(s_{1}\right)-(c / 2) w^{\prime}\left(s_{1}\right) \leqslant 0
$$

so

$$
c z-\alpha w(u-\beta)-(c / 2) z \leqslant 0 \text { at } s=s_{1} .
$$

Using the fact that $z\left(s_{1}\right)=(c / 2) w\left(s_{1}\right)$,

$$
\left(c^{2} / 4\right) w-\alpha w(u-\beta) \leqslant 0 .
$$

Dividing by $w>0$, and since $u \leqslant 1$,

$$
c^{2} / 4-\alpha(1-\beta) \leqslant 0 .
$$

This is a contradiction to the assumption $c^{2}>4 \alpha(1-\beta)$, so $z(s)>(c / 2) w(s)$ for all $s>0$ such that $u(s)>1$.

By (8) the eigenvector $x_{3}$ has $w, z$ coordinates satisfying $z=\lambda_{3} w$ and points into the region where $u<1$. Thus, solutions on $\mathscr{U}_{2}$ approaching $(1,0,0,0)$ tangent to $\mathbf{x}_{3}$ will satisfy $z>(c / 2) w$ and $u<1$ because $\lambda_{3}=\left(c+\sqrt{c^{2}-4 \alpha(1-\beta)}\right) / 2>c / 2$. This establishes the lemma.

LeMMA 6. Suppose $y(s)$ is a solution approaching $(1,0,0,0)$ tangent to $\mathbf{x}_{3}$ in the region where $u<1$ as $s \rightarrow-\infty$. Suppose further that $u(s)$ is decreasing until $\mathbf{y}(s)$ enters the region

$$
T=\{(u, v, w, z) \mid u>\beta, w>0,1-u-w>0\} .
$$

Then the solution must have $v(s)<-(c / 2) w(s)$ in $T$.

Proof. The solution approaches $(1,0,0,0)$ tangent to $\mathbf{x}_{3}$. From $(8)$ the eigenvector $\mathbf{x}_{3}$ at $(1,0,0,0)$ has $v=\lambda_{3}(u-1), w=p\left(\lambda_{3}\right)(u-1)$, where $p(\lambda)=(D-1) \lambda^{2}-$ $(1+\alpha(1-\beta))$. Then $v=u^{\prime}<0$, so $u$ is decreasing in the region $u<1$ and

$$
\begin{aligned}
1-u-w & =(1-u)-p\left(\lambda_{3}\right)(u-1)=\left(1+p\left(\lambda_{3}\right)\right)(1-u) \\
& =\left((D-1) \lambda_{3}^{2}-\alpha(1-\beta)\right)(1-u) .
\end{aligned}
$$


Recalling that $D \leqslant 1$, the expression $(D-1) \lambda_{3}^{2}-\alpha(1-\beta)$ is negative. Then in the region $u<1$, the eigenvector $x_{3}$ at $(1,0,0,0)$ lies in the region where $1-u-w<0$.

Therefore, asymptotically as $s \rightarrow-\infty$, the solution $\mathrm{y}(s)$ has $1>u>\beta, v<0$, $w>0,1-u-w<0$.

Suppose $\mathrm{y}\left(s_{1}\right) \in T, v\left(s_{1}\right) \geqslant-(c / 2) w\left(s_{1}\right)$ and $s_{1}$ is the first such value. Then $u(s) \leqslant 1$ for $s<s_{1}$ and the result of Lemma 5 shows that $z\left(s_{1}\right)>(c / 2) w\left(s_{1}\right)$. Adding the inequalities for $v\left(s_{1}\right)$ and $z\left(s_{1}\right), v\left(s_{1}\right)+z\left(s_{1}\right)>0$. Let

$$
s_{2}=\sup \left\{s<s_{1} \mid 1-u(s)-w(s) \leqslant 0\right\} \text {. }
$$

Since, asymptotically as $s \rightarrow-\infty, 1-u(s)-w(s)<0$, the value $s_{2}$ is finite. Since $1-u(s)-w(s)>0$ for $s>s_{2}$, and $1-u\left(s_{2}\right)-w\left(s_{2}\right)=0$,

$$
\left(1-u\left(s_{2}\right)-w\left(s_{2}\right)\right)^{\prime}=-v\left(s_{2}\right)-z\left(s_{2}\right) \geqslant 0 \text { or } v\left(s_{2}\right)+z\left(s_{2}\right) \leqslant 0 .
$$

Therefore the function $1-u(s)-w(s)$ has a positive maximum for some $s_{3} \in$ $\left(s_{2}, s_{1}\right)$, and $u\left(s_{3}\right)>u\left(s_{1}\right)>\beta, w\left(s_{3}\right)>0$, so $\mathrm{y}\left(s_{3}\right) \in T$. Then $1-u\left(s_{3}\right)-w\left(s_{3}\right)>$ 0 ,

$$
\begin{aligned}
& \left(1-u\left(s_{3}\right)-w\left(s_{3}\right)\right)^{\prime}=-v\left(s_{3}\right)-z\left(s_{3}\right)=0 \\
& \left(1-u\left(s_{3}\right)-w\left(s_{3}\right)\right)^{\prime \prime}=-v^{\prime}\left(s_{3}\right)-z\left(s_{3}\right) \leqslant 0 .
\end{aligned}
$$

The last may be written

$$
v^{\prime}\left(s_{3}\right)+z^{\prime}\left(s_{3}\right)=(c / D) v-(u / D)(1-u-w)+c z-\alpha w(u-\beta) \geqslant 0 .
$$

Rearranging this,

$$
c(v+z)+c(1 / D-1) v-(u / D)(1-u-w)-\alpha w(u-\beta) \geqslant 0 \text { at } s=s_{3} .
$$

Now $v+z=0$ at $s=s_{3}$ so $v\left(s_{3}\right)=-z\left(s_{3}\right)<-(c / 2) w\left(s_{3}\right)$. Therefore, $v\left(s_{3}\right)<0$. Since $D \leqslant 1,1 / D-1>0$. Finally, $1-u-w>0, u>\beta$, and $w>0$, since $\mathrm{y}\left(s_{3}\right)$ $\in T$. Therefore

$$
c(v+z)+c(1 / D-1) v-(u / D)(1-u-w)-\alpha w(u-\beta)<0 \text { at } s=s_{3} .
$$

This is a contradiction, so the assumption $v\left(s_{1}\right) \geqslant-(c / 2) w\left(s_{1}\right)$ cannot hold. This completes the proof of the lemma.

Suppose $\mathbf{y}(s)$ is a trajectory starting on $\mathscr{U}_{2}$ in the region where $u<1$, and $\mathbf{y}(s)$ is not on the 1-dimensional strongest unstable manifold $\mathscr{U}_{1}$. Then $\mathbf{y}(s)$ approaches $(1,0,0,0)$ tangent to $x_{3}$ with $u<1$. Then Lemma 6 , in connection with Lemma 5 , shows that if $\mathrm{y}(s)$ enters $T$, then $u(s)$ continues to decrease to $u=\beta$ and so enters $R$. That is, the behavior of the strongest unstable manifold in $u<1$ "continues" to nearby trajectories on $\mathscr{U}_{2}$.

The next lemma shows that there is a "last" trajectory on $\mathscr{U}_{2}$ which has $u(s)$ decreasing to the value $u=\beta$. First some notation is necessary.

A parametric representation for the 2-dimensional strongly unstable manifold $\mathscr{U}_{2}$ in a small neighborhood of $(1,0,0,0)$ is

$$
f_{2}(m, n)=(1,0,0,0)^{T}+m \mathbf{x}_{4}+n \mathbf{x}_{3}+O(|m|+|n|) .
$$

Consider a small circle on $\mathscr{U}_{2}$ parametrically given by

$$
g(\theta)=f_{2}(\varepsilon \cos (\theta+\psi), \varepsilon \sin (\theta+\psi))
$$




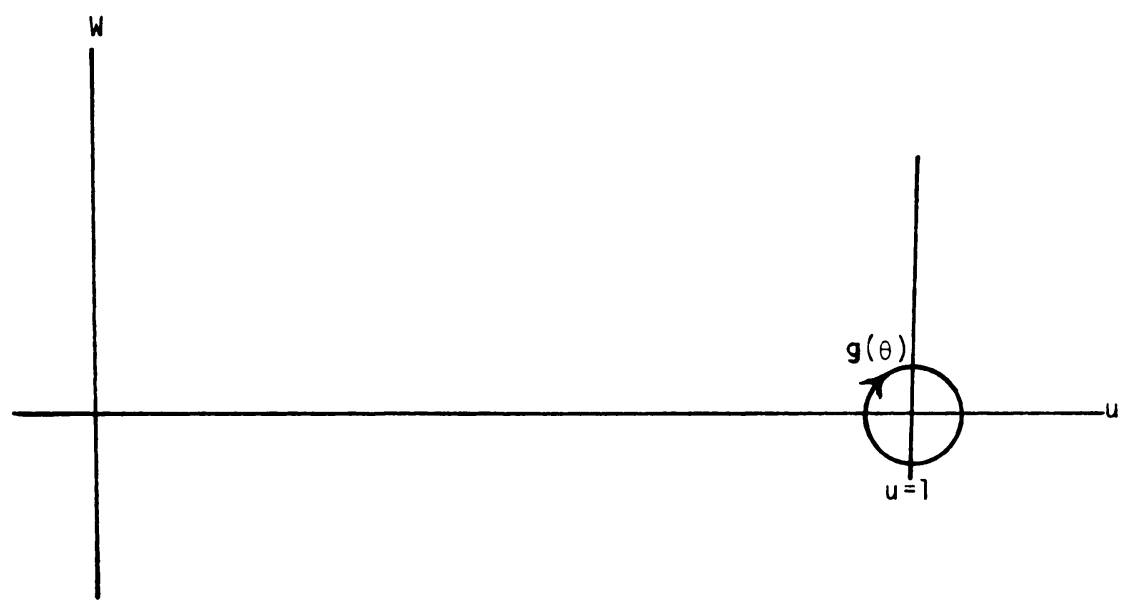

FIGURE 3. Projection of $g(\theta)$ on $\mathscr{U}_{2}$ to $u$-w plane.

The phase $\psi$ is fixed so that $g(0)$ is on $\mathscr{U}_{1}$ in the region $u<1$, and the parameter $\theta \in[0,2 \pi]$. In addition, choose $g$ so that as $\theta$ increases from 0 , the $u$ coordinate of $g(\theta)$ decreases and the $w$ coordinate increases from 0 . See Figure 3. Let $A$ be the component of the set $\left\{\theta \in[0,2 \pi] \mid\right.$ there is an $s_{0}$ such that $u\left(s_{0}, g(\theta)\right)=\beta$ and $v(s, g(\theta)) \leqslant 0$ for $\left.-\infty<s \leqslant s_{0}\right\}$ containing 0 . Then Lemma 3 shows that $0 \in A$, so $A$ is not empty. Lemma 4 shows that $A$ is bounded above by a value less than $2 \pi$. Let $\theta_{1}=\sup A$ and $\mathrm{y}_{1}=g\left(\theta_{1}\right)$.

LEMmA 7. There is an $s_{0}$ such that $u\left(s_{0}, \mathbf{y}_{1}\right)=\beta, w\left(s_{0}, \mathbf{y}_{1}\right)>1-\beta, v\left(s_{0}, \mathbf{y}_{1}\right)=0$.

Remark. This lemma shows that the "last" trajectory $\mathbf{y}\left(s, \mathbf{y}_{1}\right)$ to attain the value $u=\beta$ just grazes the region $P$ and enters the region $S$. Figure 4 is a projection of this situation to the $u-w$ plane.

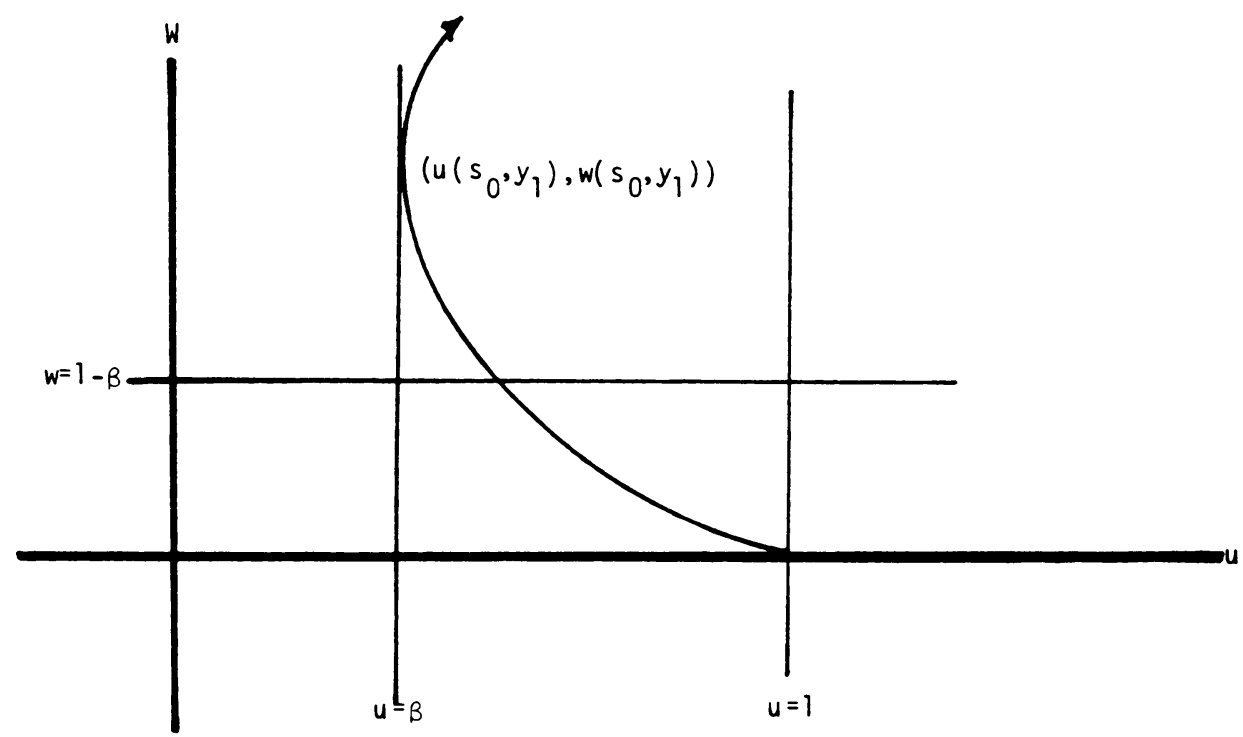

Figure 4. Projection to the $u-w$ plane of the trajectory described in Lemma 7. 
Proof. Note that since $g(0)$ is on $\mathscr{U}_{1}$ with $u<1$, Lemma 3 shows that if $u\left(s_{0}, g(0)\right)=\beta$ then $v\left(s_{0}, g(0)\right)=(d / d s) u\left(s_{0}, g(0)\right)<0$. Therefore $u(s, g(\theta))=\beta$ for $s=s_{0}(\theta)$ for $\theta$ in a small neighborhood of $\theta=0$. Therefore $\theta_{1} \neq 0$.

If $g\left(\theta^{*}\right)$ is on the branch of $\mathscr{U}_{1}$ in the region where $u>1$, then $u\left(s, g\left(\theta^{*}\right)\right)>1$, as shown by Lemma 4 . Therefore $\theta_{1}<\theta^{*}$. Therefore, the trajectory of $\mathbf{y}\left(s, \mathbf{y}_{1}\right)$ approaches $(1,0,0,0)$ tangent to $\mathbf{x}_{3}$.

Several cases could occur for $\mathbf{y}\left(s, \mathbf{y}_{1}\right)$.

1. It is not possible for the solution $\mathbf{y}\left(s, \mathbf{y}_{1}\right)$ to remain in the region where $\beta<u<1,0<w<1-\beta$, for all $s>0$. This follows from Lemma 5 , since in this case $w^{\prime}=z>(c / 2) w>0$ for all $s>0$, and $w$ could not be bounded.

2. It is not possible that there is an $s_{1}$ such that $v\left(s_{1}, \mathbf{y}_{1}\right)=0, \beta<u\left(s_{1}, \mathbf{y}_{1}\right)<1$, $w\left(s_{1}, y_{1}\right)>0$. Suppose that it did occur for some $s_{1}$. Lemma 6 shows that in the region $T, v(s)<-(c / 2) w(s)<0$, so it must be, then, that $1-u\left(s_{1}\right)-w\left(s_{1}\right) \leqslant 0$, $\beta<u\left(s_{1}\right)<1, w\left(s_{1}\right)>0$. If $1-u\left(s_{1}\right)-w\left(s_{1}\right)=0, v\left(s_{1}\right)=0, w\left(s_{1}\right)>0$, then $z\left(s_{1}\right)$ $>(c / 2) w\left(s_{1}\right)>0$, so, following the trajectory backwards a short time, the trajectory is in $T$. In fact, there is a $\delta>0$ such that if $s \in\left(s_{1}-\delta, s_{1}\right)$, then $\mathbf{y}(s)$ is in $T$, $w\left(s_{1}\right)-w(s)<\frac{1}{4} w\left(s_{1}\right),|v(s)|<(c / 4) w\left(s_{1}\right)$. Then $\frac{3}{4} w\left(s_{1}\right)<w(s),-(c / 4) w\left(s_{1}\right)<$ $v(s)$. Rearranging these, we see that $\mathrm{y}(s)$ is in $T$ and $-(c / 3) w(s)<v(s)$, which is a contradiction to Lemma 6.

We conclude that if there is an $s_{1}$ such that $v\left(s_{1}\right)=0$, then $1-u\left(s_{1}\right)-w\left(s_{1}\right)<0$. Then

$$
v^{\prime}\left(s_{1}\right)=-(1 / D) u\left(s_{1}\right)\left(1-u\left(s_{1}\right)-w\left(s_{1}\right)\right)>0,
$$

so $v$ increases. The assumption is that $v\left(s, \mathbf{y}_{1}\right)=0, u\left(s_{1}, \mathbf{y}_{1}\right)>\beta$. The previous sentence shows that $v^{\prime}\left(s_{1}, \mathbf{y}_{1}\right)>0$. The Implicit Function Theorem and continuity of solutions in initial conditions then shows that for all $\theta$ in a sufficiently small neighborhood of $\theta_{1}$, there is an $s_{1}=s_{1}(\theta)$ such that $v\left(s_{1}(\theta), g(\theta)\right)=0$, $u\left(s_{1}(\theta), g(\theta)\right)>\beta$, and, since this cannot occur in the region $T$, we must have

$$
1-u\left(s_{1}(\theta)\right)-w\left(s_{1}(\theta)\right)<0 .
$$

Then $v^{\prime}\left(s_{1}(\theta), g(\theta)\right)>0$, so for $\theta<\theta_{1}$ and in this small neighborhood of $\theta_{1}$, we have violated the requirement that $v(s)$ be nonpositive until $u(s)=\beta$. This contradicts the definition of $\theta_{1}$. Thus, this possibility is ruled out.

3. The next case to be covered is to rule out the possibility that $u\left(s, \mathbf{y}_{1}\right)>\beta$ for all $s$. We know from part 2 that $u(s)$ is decreasing, and from Lemma 5 that $w(s)$ is increasing. From part 1 we know that $w(s)$ cannot be bounded above. Thus, under the assumption that $u\left(s, \mathrm{y}_{1}\right)>\beta$ for all $s$, we have that $1-u(s)-w(s)$ decreases without bound.

Let $K>\lambda_{4}$. Since $\mathbf{y}\left(s, \mathbf{y}_{1}\right)$ approaches $(1,0,0,0)$ tangent to $\mathbf{x}_{3}$ as $s \rightarrow-\infty$, we may rescale the time if necessary to see that

$$
v(0)>K(u(0)-1) .
$$

If for some first $s_{1}, v\left(s_{1}\right)=K\left(u\left(s_{1}\right)-1\right)$, then $v^{\prime}\left(s_{1}\right)-K u^{\prime}\left(s_{1}\right) \leqslant 0$. Substituting from (5),

$$
(c / D) v-(u / D)(1-u-w)-K v \leqslant 0 \text { at } s=s_{1} \text {. }
$$


Then using the equation $v\left(s_{1}\right)=K\left(u\left(s_{1}\right)-1\right)$,

$$
\left(-K^{2}+(c / D) K+u / D\right)(u-1)+u w / D \leqslant 0 \text { at } s=s_{1} .
$$

Now $u\left(s_{1}\right)<1$ so

$$
-K^{2}+(C / D) K+u / D<-K^{2}+(C / D) K+1 / D \text { at } s=s_{1} .
$$

Since $K>\lambda_{4},-K^{2}+(c / D) K+1 / D<0, \beta<u\left(s_{1}\right)<1$, and $w\left(s_{1}\right)>0$. Therefore

$$
\left(-K^{2}+(c / D) K+u / D\right)(u-1)+u w / D>0 \text { at } s=s_{1} .
$$

This is a contradiction so we must have

$$
v(s)>K(u(s)-1) \text { for all } s .
$$

This means that $v$ is bounded below by $K(\beta-1)$. Then

$$
v^{\prime}=(c / D) v-(u / D)(1-u-w)
$$

is increasing without bound since $(1-u-w)$ decreases without bound. But then $v$ does not remain negative so $u$ is not decreasing, and we are back to part 2. This contradiction shows that we cannot have the trajectory $\mathbf{y}\left(s, \mathbf{y}_{1}\right)$ with $\beta<u(s)<1$ and $w(s)$ increasing without bound.

4. By the results of part 2 we know that $v(s, g(\theta))<0$ as long as $u(s, g(\theta)) \geqslant \beta$. It cannot be that there is an $s_{0}$ such that $u\left(s_{0}, \mathbf{y}_{1}\right)=\beta, w\left(s_{0}, \mathbf{y}_{1}\right)<1-\beta$, because should this occur, Lemma 6 shows that $v\left(s_{0}, \mathbf{y}_{1}\right)<0$. Then for all $\theta$ sufficiently near $\theta_{1}$, there is an $s_{0}$ such that $u\left(s_{0}, g(\theta)\right)=\beta$. This is true in particular for $\theta>\theta_{1}$. This contradicts the definition of $\theta_{1}$.

5. It cannot be that there is an $s_{0}$ such that $u\left(s_{0}, \mathbf{y}_{1}\right)=\beta, w\left(s_{0}, \mathbf{y}_{1}\right)=1-\beta$. As in part 4 , if $v\left(s_{0}, \mathbf{y}_{1}\right)<0$ then we have a contradiction to the definition of $\theta_{1}$. If $v\left(s_{0}, \mathbf{y}_{1}\right)=0$ then $z\left(s_{0}, \mathbf{y}_{1}\right)>0$ by Lemma 5 , and by following the trajectory backwards, we will be in the region $T$. Then the same argument as in part 2 yields a contradiction to Lemma 6.

6. The only possibility remaining is the conclusion of the lemma, and the proof is complete.

We remark that parts 1,2 and 3 actually show that solutions starting from $g(\theta)$, $0<\theta<\theta_{1}$, cannot have $u(s)>\beta$ for all $s>0$. Parts 4 and 5 show that when these solutions $\mathrm{y}(s, g(\theta)), 0<\theta<\theta_{1}$, cross $u=\beta$, they do so transversally. This also follows from the analysis of the exit set, since trajectories touching $u=\beta$ with $v<0$ or $z>0$ exit immediately into $R$ or $P$. Thus, solutions starting from the arc $g(\theta)$, $0<\theta<\theta_{1}$, on $\mathscr{U}_{2}$ enter either region $R$ or region $P$. The $\operatorname{arc} g(\theta), 0 \leqslant \theta<\theta_{1}$, provides one side of the quadrilateral $\Sigma$.

D. We now define the next side of the quadrilateral $\Sigma$. Solutions starting from this side enter the region $S$. The analysis is considerably simpler since it is mostly local analysis and uses only the Intermediate Value Theorem and the Implicit Function Theorem.

The second side of the quadrilateral is composed of two portions. One portion is an $\operatorname{arc} g(\theta), \theta_{1} \leqslant \theta \leqslant \theta_{2}$, where the $v$ coordinate of $g\left(\theta_{2}\right)$ is 0 ; the second is an arc of the circle of intersection of a small sphere surrounding $(1,0,0,0)$ in $\mathscr{U}_{3}$ and the hyperplane defined by $v=0$. See Figure 5 . 


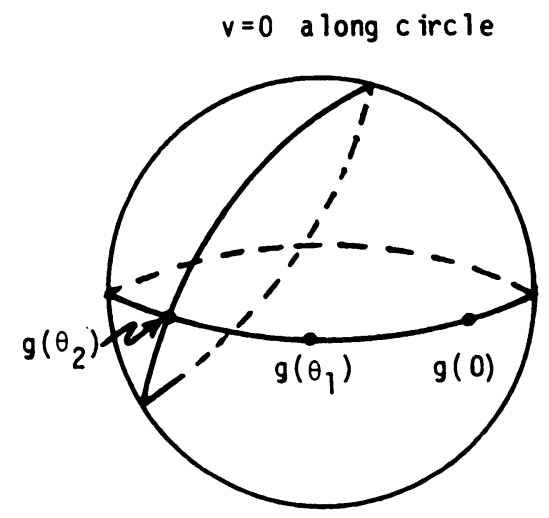

FIGURE 5. Diagram of second side of quadrilateral $\Sigma$.

We first locate the value $\theta_{2}$.

LEMMA 8. There is a value $\theta_{2}$ such that the $v$ coordinate of $g\left(\theta_{2}\right)$ is zero. Also $\theta_{2}>\theta_{1}$.

Proof. Since $g(0) \in \mathscr{U}_{1}$ in the region $u<1$, the $v$ coordinate of $g(0)$ is negative. There is a $\theta^{*}, 0<\theta^{*}<2 \pi$, such that $g\left(\theta^{*}\right)$ is on $\mathscr{U}_{1}$, with $u>1$. The $v$ coordinate of $g\left(\theta^{*}\right)$ is positive. Then there is a $\theta_{2}>0$ such that the $v$ coordinate of $g\left(\theta_{2}\right)$ is zero. The proof of Lemma 7, part 2, says that for $s \geqslant 0$ the trajectory through $g\left(\theta_{1}\right)$ can never have a $v$ coordinate equal to 0 if $u>\beta$. Therefore $\theta_{2}>\theta_{1}$. This completes the proof.

A parametric representation for the 3-dimensional unstable manifold $\mathscr{U}_{3}$ in a neighborhood of $(1,0,0,0)$ is

$$
f_{3}(m, n, p)=(1,0,0,0)^{T}+m \mathbf{x}_{4}+n \mathbf{x}_{3}+p \mathbf{x}_{2}+O(|m|+|n|+|p|) .
$$

Consider the function

$$
h(\theta, \phi)=f_{3}(\varepsilon \cos \theta \sin \phi, \varepsilon \sin \theta \sin \phi, \varepsilon \cos \phi)
$$

for $\theta \in[0,2 \pi), \phi \in[0, \pi]$. Then the image of $h$ is a small sphere surrounding $(1,0$, $0,0)$ in $\mathscr{U}_{3}$. Note also that this sphere contains the $\operatorname{arc} g(\theta)=h(\theta, \pi / 2)$.

From the previous lemma we know that the sphere intersects the hyperplane defined by $v=0$ in at least one point $h\left(\theta_{2}, \pi / 2\right)$. The next lemma shows that this intersection actually occurs along a smooth curve.

LEMMA 9. The sphere intersects the hyperplane defined by $v=0$ in a smooth closed curve.

Proof. We want to show that there is a $C^{1}$ function $\phi(\theta)$ for $\theta \in[0,2 \pi]$, with $\phi(\theta) \in[0, \pi]$, such that the $v$ coordinate of $h(\theta, \phi(\theta))$ is zero. That is, using (8) and (9),

$$
0=-\varepsilon \lambda_{4} \cos \theta \sin \phi(\theta)-\varepsilon \lambda_{3} \sin \theta \sin \phi(\theta)-\varepsilon \lambda_{2} \cos \phi(\theta)+O(\varepsilon)
$$


for some $\phi(\theta) \in C^{1}$. From Lemma 8 we know that if $\theta=\theta_{2}, \phi=\pi / 2$ then (10) is satisfied. We use the Implicit Function Theorem to extend this to a smooth function in a neighborhood of $\left(\theta_{2}, \pi / 2\right)$. This neighborhood and function can continue to be extended as long as the hypotheses of the Implicit Function Theorem continue to hold.

For simplicity, divide (10) by $-\varepsilon$ on both sides. Let

$$
K(\theta, \phi)=\lambda_{4} \cos \theta \sin \phi+\lambda_{3} \sin \theta \sin \phi+\lambda_{2} \cos \phi+O(1) .
$$

Then

$$
\partial K / \partial \phi=\lambda_{4} \cos \theta \cos \phi+\lambda_{3} \sin \theta \cos \phi-\lambda_{2} \sin \phi+O(1) .
$$

We want to show $\partial K / \partial \phi \neq 0$. By taking $\varepsilon$ small enough, this will be possible if

$$
\lambda_{4} \cos \theta \cos \phi+\lambda_{3} \sin \theta \cos \phi-\lambda_{2} \sin \phi \neq 0 .
$$

At $\left(\theta_{2}, \pi / 2\right), \partial K / \partial \phi=-\lambda_{2}$, so the Implicit Function Theorem applies.

For $\varepsilon$ sufficiently small, $\theta$ and $\phi$, satisfying (10), will lie in a neighborhood of the curve defined by

$$
\lambda_{4} \cos \theta \sin \phi+\lambda_{3} \sin \theta \sin \phi+\lambda_{2} \cos \phi=0,
$$

or, equivalently,

$$
\cot \phi=\left(\lambda_{4} \cos \theta+\lambda_{3} \sin \theta\right) /-\lambda_{2} .
$$

The points where $\partial K / \partial \phi=0$ are in a neighborhood of the curve defined by

$$
\lambda_{4} \cos \theta \cos \phi+\lambda_{3} \sin \theta \cos \phi-\lambda_{2} \sin \phi=0
$$

or, equivalently,

$$
\cot \phi=\lambda_{2} /\left(\lambda_{4} \cos \theta+\lambda_{3} \sin \theta\right) .
$$

Then it is easy to see that the curves given by (11) and (12) are uniformly bounded away from each other. The neighborhoods of these curves are then disjoint for $\varepsilon$ sufficiently small. Thus, the $\theta$ on the sphere given by $\phi(\theta)$ may be extended to a smooth closed curve on the sphere, completing the proof.

We next define the third side of the quadrilateral $\Sigma$. Solutions starting from this side enter the region $Q$. This side is the intersection of the sphere defined by $h(\theta, \phi)$ with the hyperplane defined by $z=0$. The proof that such a smooth intersection curve exists is similar to the previous proof.

LEMMA 10. The sphere intersects the hyperplane defined by $z=0$ in a smooth closed curve.

Proof. Using (8) and (9) we want to show there is a $C^{1}$ function $\phi(\theta)$ for $\theta \in[0,2 \pi]$, with $\phi(\theta)=[0, \pi]$, such that

$$
0=-\varepsilon \lambda_{3} p\left(\lambda_{3}\right) \sin \theta \sin \phi(\theta)-\varepsilon \lambda_{2} p\left(\lambda_{2}\right) \cos \phi(\theta)+O(\varepsilon) .
$$

The remarks preceding Lemma 3 show that $\mathscr{U}_{1}$ lies in the hyperplane defined by $z=0$. Then the point $\theta=0, \phi=\pi / 2$, which is on $\mathscr{U}_{1}$ by the choice made in the remarks previous to Lemma 7 , satisfies (13).

Divide (13) by $-\varepsilon$ on both sides. Let

$$
l(\theta, \phi)=\lambda_{3} p\left(\lambda_{3}\right) \sin \theta \sin \phi+\lambda_{2} p\left(\lambda_{2}\right) \cos \phi+O(1) .
$$


Then

$$
\partial l / \partial \phi=\lambda_{3} p\left(\lambda_{3}\right) \sin \theta \cos \phi-\lambda_{2} p\left(\lambda_{2}\right) \sin \phi+O(1) .
$$

Then for $\varepsilon$ sufficiently small, $\partial l / \partial \phi \neq 0$, provided

$$
\lambda_{3} p\left(\lambda_{3}\right) \sin \theta \cos \phi-\lambda_{2} p\left(\lambda_{2}\right) \sin \phi \neq 0 .
$$

For $\theta=0, \phi=\pi / 2$, this last expression is $-\lambda_{2} p\left(\lambda_{2}\right) \neq 0$, so the Implicit Function Theorem applies.

For $\varepsilon$ sufficiently small, $\theta$ and $\phi$, satisfying (13), will be in a neighborhood of the curve defined by

$$
\cot \phi=\left(\lambda_{3} p\left(\lambda_{3}\right) / \lambda_{2} p\left(\lambda_{2}\right)\right) \sin \theta .
$$

The points where $\partial l / \partial \phi=0$ are in a neighborhood of the curve defined by

$$
\cot \phi=\lambda_{2} p\left(\lambda_{2}\right) / \lambda_{3} p\left(\lambda_{3}\right) \sin \theta \text {. }
$$

Again it is easily seen that the curves given by (14) and (15) are uniformly bounded away from each other. Then the neighborhoods of these curves are disjoint for sufficiently small $\varepsilon$. Then the curve given by $\phi(\theta)$ may be extended to a smooth closed curve on the sphere given by $h(\theta, \phi)$, completing the proof.

We next show that the curves given by Lemmas 9 and 10 intersect.

LEMMA 11. There is a point, say $y_{3}$, on the sphere such that the $v$ and $z$ coordinates of $y_{3}$ are both zero.

Proof. For $\theta=0, \phi=\pi / 2$, we are on the intersection of the sphere and the hyperplane defined by $z=0$. We are also on the branch of $\mathscr{U}_{1}$ in the region $u<1$. The tangency of $\mathscr{U}_{1}$ to $\mathbf{x}_{4}$ shows that this point has $v<0$.

For $\theta=\theta^{*}, \phi=\pi / 2$, we are also on the intersection of the sphere and the hyperplane defined by $z=0$ because we are on the branch of $\mathscr{U}_{1}$ contained in the region $u>1$. As used in the proof of Lemma 4 this point has $v>0$.

Following the intersection of the sphere and the hyperplane defined by $z=0$ given by the function in Lemma 10 from $\theta=0$ to $\theta=\theta^{*}$, there is some $\theta_{3}, \phi\left(\theta_{3}\right)$, such that the point $h\left(\theta_{3}, \phi\left(\theta_{3}\right)\right)$ has $v$ coordinate zero. This completes the proof.

It will be convenient to have some notation for the points obtained in this section. Choose a small enough neighborhood of $(1,0,0,0)$ so that the conditions of tangency required in Lemmas 1-6 are satisfied. Fix $\varepsilon$ sufficiently small so that the sphere given by the function $h(\theta, \phi)$ is contained within this neighborhood. The value of $\varepsilon$ should also be small enough to assure the conditions of Lemmas 9 and 10 are satisfied. Let $y_{0}$ be the point of intersection of the strongest unstable manifold $\mathscr{U}_{1}$ and the sphere given by $h(\theta, \phi)$. In the previous notation $y_{0}=g(0)$. As before, $y_{1}=g\left(\theta_{1}\right)$ is the initial point of the solution which just grazes the region $P$. This point $y_{1}$ is also on the sphere; in fact it is on the curve of intersection of the manifold $\mathscr{U}_{2}$ and the sphere. Let $y_{2}=g\left(\theta_{2}\right)$ as in Lemma 8 . Let $y_{3}$ be the point of the sphere with both its $v$ and $z$ coordinates equal to zero. The point $\mathbf{y}_{3}$ is common to both the intersection of the sphere with the hyperplane defined by $v=0$ and the intersection of the sphere with the hyperplane defined by $z=0$.

We now have a topological triangle defined on the sphere. One corner is the point $\mathbf{y}_{0}$ and a side is the arc given by $g(\theta)$ from $\theta=0$ to $\theta=\theta_{1}$. This side terminates in 


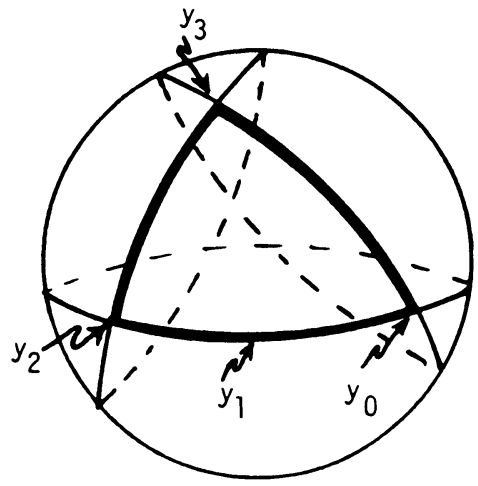

FIGURE 6. Diagram of topological triangle on sphere.

the corner point $y_{1}$. The next side is in two portions which are connected at the point $y_{2}$. The first is the arc of $g(\theta)$ from $\theta=\theta_{1}$ to $\theta=\theta_{2}$, an arc from the corner point $y_{1}$ to the point $y_{2}$. The second is the arc of the curve of intersection of the sphere with the plane defined by $v=0$ from the point $\mathbf{y}_{2}$ to the corner $\mathbf{y}_{3}$. The last side of this topological triangle is the arc of the curve of intersection of the sphere with the hyperplane defined by $z=0$ from the corner point $y_{3}$ to the corner point $y_{0}$. See Figure 6.

This triangle is almost the set $\Sigma$ we desire. However this triangle does not satisfy requirement (2) of Proposition 2 for the following reason. The solutions of (5) starting from the point $\mathrm{y}_{0}$ will have $w \equiv 0, z \equiv 0$ because such solutions start on the strongest unstable manifold $\mathscr{U}_{1}$ contained in $\mathscr{V}$. Let $\mathbf{y}\left(s, \mathbf{y}_{0}\right)$ be such a solution. From Lemma 3 there is an $s_{0}$ such that $u\left(s_{0}, \mathbf{y}_{0}\right) \equiv \beta, v\left(s_{0}, \mathbf{y}_{0}\right)<0$. That is, $\mathbf{y}\left(s, \mathbf{y}_{0}\right)$ hits the boundary of $R$ at a finite time $s_{0}$. For $0 \leqslant s<s_{0}$, the solution has $u\left(s, \mathbf{y}_{0}\right)>\beta, w(s)=0, z(s)=0$. Therefore we have a small neighborhood in $\mathbf{R}^{4}$ around the point $\mathbf{y}\left(s, \mathbf{y}_{0}\right)$ which contains points, with $u>\beta, w>0, z=0$, which are in $W^{-}$.

Let $U$ be a small neighborhood in $\mathbf{R}^{4}$ around the point $\mathbf{y}_{0}$. Let $U$ be small enough that it does not contain $\mathbf{y}_{1}$ or $\mathbf{y}_{3}$. Also let $U$ be small enough that if $\mathbf{y}^{*} \in U$, then there is an $s_{0}\left(\mathbf{y}^{*}\right)$ such that $u\left(s_{0}\left(\mathbf{y}^{*}\right), \mathbf{y}^{*}\right)=\beta$. Recall that $\mathbf{y}\left(s, \mathbf{y}_{0}\right)$ crosses the hyperplane defined by $u=\beta$ transversally at $s=s_{0}$, so this is possible. Let $V$ be a small ball in $\mathbf{R}^{4}$ centered at $\mathbf{y}_{0}$ contained in $U$. Consider the curve of intersection of the sphere $\partial V$ with the sphere in $\mathscr{U}_{3}$ defined by $h(\theta, \phi)$. This curve is the fourth and final side of the quadrilateral $\Sigma$.

Let $\mathbf{y}_{4}$ be the intersection of $\partial V$ with the arc of intersection of the sphere defined by $h(\theta, \phi)$ and the hyperplane defined by $z=0$. That is, $y_{4}$ is the corner between the third and fourth sides of $\Sigma$. Let $y_{5}$ be the intersection of $\partial V$ with the curve defined by $g(\theta)$. That is, $y_{5}$ is the corner between the fourth and first sides of $\Sigma$ given by $g(\theta)$. See Figure 7.

The construction of $\Sigma$ gives us information about the images of the sides under the exit time mapping $F(y)=y \cdot T(y)$. The first side is carried by the flow of the 
differential equation into either region $R$ or region $P$, as shown by the first five lemmas. The second side is carried by the flow into the region $S$. The proof of Lemma 5 , parts $1,2,3$, shows that the first portion of the second side has $u>\beta$, $v=0,1-u-w<0$ for some $s_{0}>0$ and so enters $S$. The exit set analysis shows that points on the intersection of the sphere with $v=0$ enter $S$ immediately. The third side is carried by the flow immediately into the region $Q$ for a similar reason. The fourth side at least exits into $R$. Points on the fourth side near the corner $y_{4}$ may exit first into $Q$. In any case, points on the fourth side are carried into regions $Q$ or $R$.

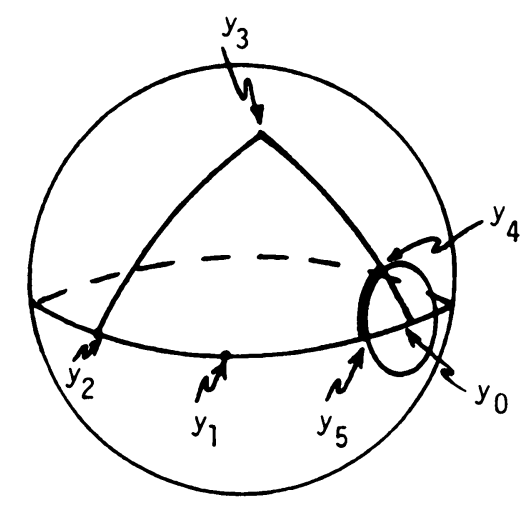

FIgURE 7. Construction of the fourth side of quadrilateral $\Sigma$.

Thus the boundary of the quadrilateral $\Sigma$ is carried by the flow into a closed curve on the boundary of $W$ surrounding the point $(\beta, 0,1-\beta, 0)$. See Figures 8 and 9 for projections of this situation into the $u-w-v$ subspace and the $u-w-z$ subspace.

E. Using the information from the previous two subsections, we can use Proposition 2 to assert the existence of a solution remaining in $W$ for all $s$. We will then show that this solution is bounded. Then the Invariance Principle will show that this solution approaches $(\beta, 0,1-\beta, 0)$ as $s \rightarrow \infty$. This solution is then a solution of (5) satisfying boundary conditions (4).

LEMMA 12. There is a $\mathbf{y}^{*} \in \Sigma$ such that the solution $\mathbf{y}\left(s, \mathbf{y}^{*}\right)$ remains in the region $W$ for all $s$; that is, $\Sigma \neq \Sigma^{0}$.

Proof. The set $W$ is closed. Therefore condition (1) of Theorem 2.1 is satisfied trivially.

Suppose $\mathbf{y}_{0} \in \Sigma, s<T\left(\mathbf{y}_{0}\right), \mathbf{y}\left(s ; \mathbf{y}_{0}\right) \in W$ and $\mathbf{y}\left(s, \mathbf{y}_{0}\right) \notin W^{-}$. Because $W^{-} \subseteq \partial W$, we must have either $\mathrm{y}\left(s ; \mathbf{y}_{0}\right) \in$ int $W$ or $\mathbf{y}\left(s, \mathbf{y}_{0}\right) \in \partial W / W^{-}$. If $\mathbf{y}\left(s, \mathbf{y}_{0}\right) \in$ int $W$, then trivially there is an open set $U$ around $\mathbf{y}\left(s, \mathbf{y}_{0}\right)$ disjoint from $\partial W$.

If $\mathbf{y}\left(s, \mathbf{y}_{0}\right) \in \partial W \backslash W^{-}$there are several cases to eliminate. Since $\mathbf{y}\left(s, \mathbf{y}_{0}\right)$ is near $(1,0,0,0)$ at $s=0$, it is not possible that $\mathbf{y}\left(s, \mathbf{y}_{0}\right) \in \mathscr{W}$, where $\mathscr{W}$ is the invariant manifold defined by $u=v=0$. 


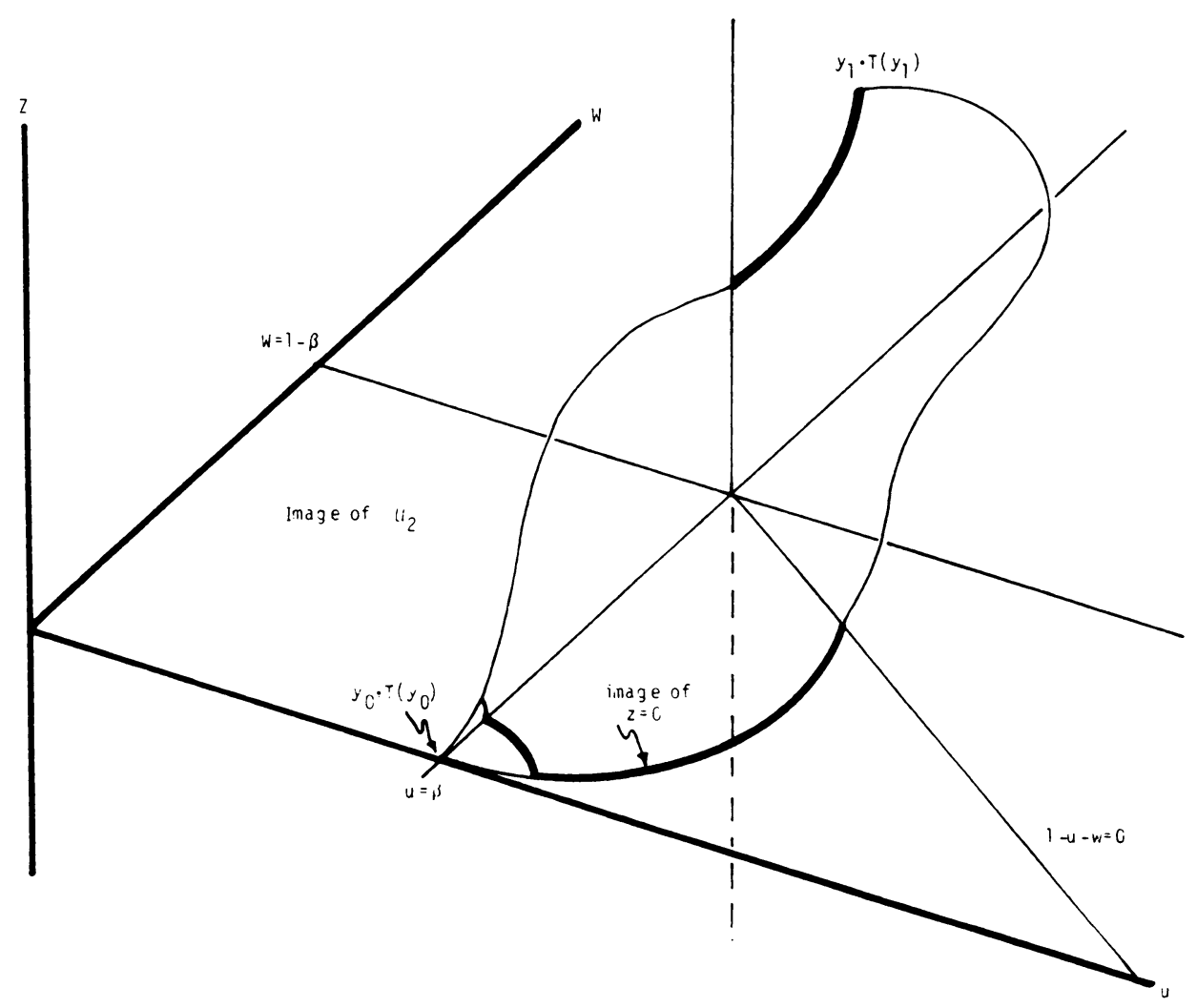

FIGURE 8. Projection into $u-w-z$ space of image of $\partial \Sigma$ on $W^{-}$.

By construction, $\Sigma \cap \mathscr{V}=\varnothing$, where $\mathscr{V}$ is the invariant manifold defined by $\boldsymbol{w}^{\prime}=\boldsymbol{z}=0$. Therefore, $\mathbf{y}\left(s, \mathbf{y}_{0}\right)$ is not in $\mathscr{V}$.

If $\mathbf{y}\left(s ; \mathbf{y}_{0}\right)$ is in the portion of $\partial W \backslash W^{-}$with $u>\beta, w<0, z=0$, then, since $z^{\prime}=c z-\alpha w(u-\beta)>0$, the trajectory was previously in the region $Q$. This is contrary to the assumption that $s<T\left(\mathbf{y}_{0}\right)$.

If $\mathbf{y}\left(s ; \mathbf{y}_{0}\right)$ is in the portion $\partial W \backslash W^{-}$with $u<0,1-u-w>0$, and $v=0$, then, since $v^{\prime}=c v-u(1-u-w)>0$, the solution $\mathbf{y}(s)$ was previously in the region $R$. This is contrary to the assumption that $s<T\left(\mathbf{y}_{0}\right)$.

Note that, by construction, $\Sigma$ is compact, intersects each trajectory only once, and is simply connected.

If $\Sigma=\Sigma^{0}$ then there would be a homeomorphism between $\Sigma$ and its image on $W^{-}$. However, in Appendix II we prove that $W^{-}$is not simply connected. In fact, we show that a set $V$ containing $W^{-}$is not simply connected. $V$ is defined as

$$
V=(\bar{P} \cup \bar{Q} \cup \bar{R} \cup \bar{S}) \backslash\{(\beta, 0,1-\beta, 0)\} .
$$

The exit time mapping from $\Sigma$ to (a subset of the boundary of) $V$ cannot be a homeomorphism because the exit time mapping carries the boundary of the quadrilateral $\Sigma$ to a loop in (a subset of the boundary of) $V$ surrounding the "hole" which 
is the removed point $(\beta, 0,1-\beta, 0)$. Except for the purpose of showing that a homeomorphism from $\Sigma$ to $W^{-}$cannot exist, it seems preferable not to mention $V$. The proof of existence of traveling wave solutions is more easily motivated and explained in terms of $W, W^{-}, P, Q, R$, and $S$.

An alternate way of viewing this is as follows: Suppose $\Sigma=\Sigma^{0}$. Then the exit time mapping would be a homotopy from the $\Sigma$ to its image on $W^{-}$. That is, we would have a homotopy of $\Sigma$ to $V$. In Appendix II we exhibit a homotopy from $V$ to a copy of $\mathbf{R}^{3}$ with a line removed, so that $V$ is not simply connected. Applying this homotopy of $V$ to the image of $\Sigma$ under the exit time mapping, we see that the image is not simply connected. Showing that the image of $\Sigma$ under the exit time mapping is not simply connected depends crucially on the fact that the image of the boundary traverses the boundaries of $R, P, Q, S$, back to $R$ in that order.

Thus $\Sigma \neq \Sigma^{0}$ and there is some $\mathbf{y}^{*} \in \Sigma$ such that $\mathbf{y}\left(s, \mathbf{y}^{*}\right) \in W$ for all $s$. This completes the lemma.

For convenience let $\mathbf{y}_{1}(s)$ denote the solution $\mathbf{y}\left(s, \mathbf{y}^{*}\right)$. Let the coordinate functions of $y_{1}(s)$ be $u_{1}(s), v_{1}(s), w_{1}(s), z_{1}(s)$.

LEMMA 13. The coordinate functions $u_{1}(s)$ and $w_{1}(s)$ are positive for all time.

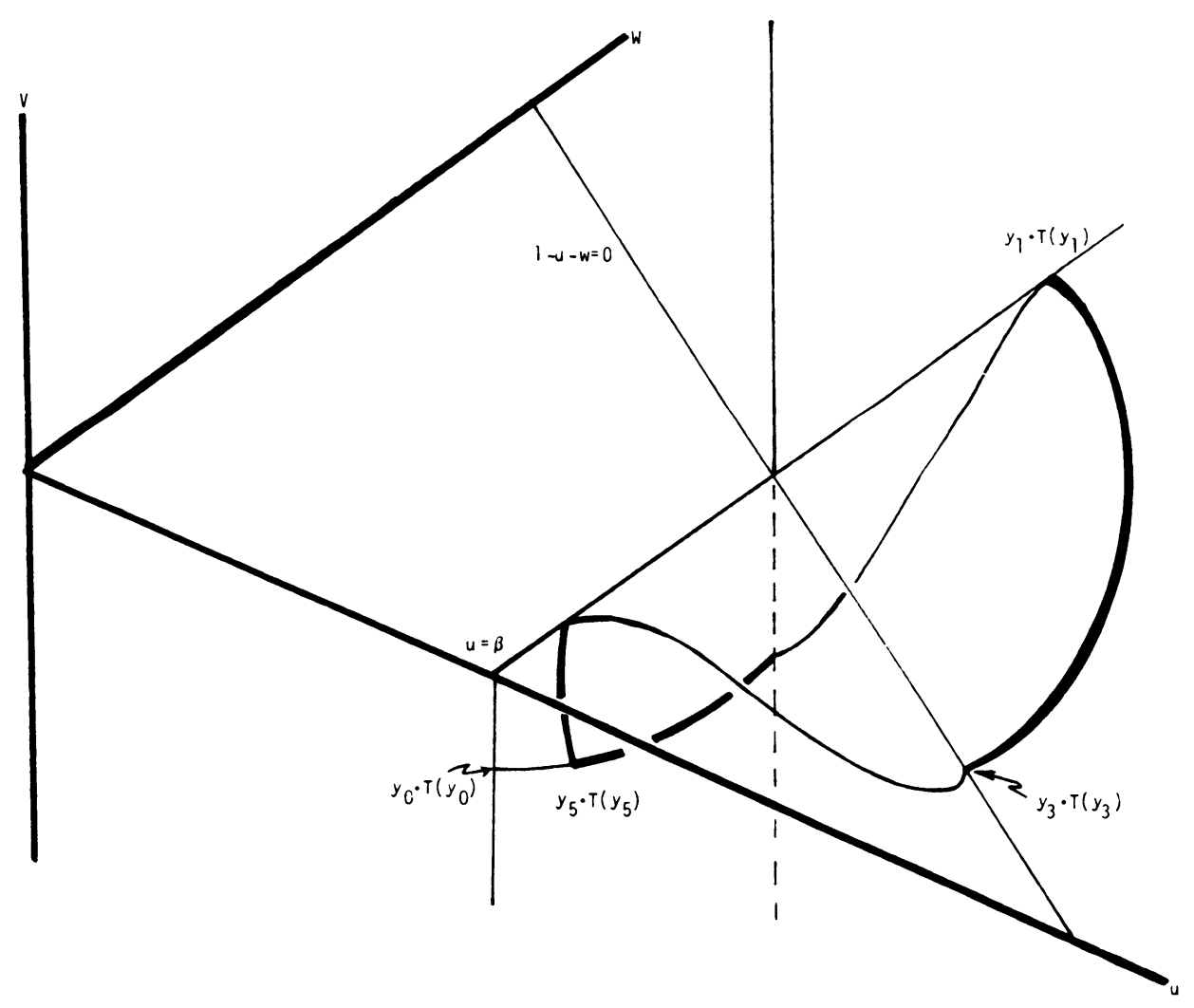

FIGURE 9. Projection into $u-w-v$ space of image of $\partial \Sigma$ on $W^{-}$. 
Proof. The solution $y_{1}(s)$ approaches $(1,0,0,0)$ on $\mathscr{U}_{3}$ as $s \rightarrow-\infty$ in the region $u<1$, by construction. Therefore, we may say that $1>u_{1}(s)>0$ and, using (8), we see $w_{1}(s)>0$ for all $s<0$.

Suppose $s_{1}$ is the first $s$ such that $u_{1}(s)=0$. Because $s_{1}$ is the first time, $v_{1}\left(s_{1}\right)=u_{1}^{\prime}\left(s_{1}\right) \leqslant 0$. Since $\mathbf{y}_{1}(s) \notin \mathscr{W}, v_{1}\left(s_{1}\right)<0$. Since $\mathbf{y}_{1}(s) \notin R, w_{1}\left(s_{1}\right)>1$. Since $w_{1}\left(s_{1}\right)>1-\beta$ and $\mathbf{y}_{1}\left(s_{1}\right) \notin P, z_{1}\left(s_{1}\right)<0$.

Therefore $u_{1}\left(s_{1}\right)$ will decrease into the region where $u<0$ and $1-u-w<0$. Since $v^{\prime}=(c / D) v-(u / D)(1-u-w)$, the quantity $v(s)$ will continue to decrease, so $u^{\prime}(s)$ will be bounded above by $v\left(s_{1}\right)$ for $s$ such that $1-u_{1}(s)-w_{1}(s)<0$.

Also, if $u_{1}(s)<0,1-u_{1}(s)-w_{1}(s)<0$ then $w_{1}(s)>1>1-\beta$, so we must have $z_{1}(s)<0$. Thus the quantity $v_{1}(s)+z_{1}(s)$ will be bounded above by $v\left(s_{1}\right)<0$. Thus, $1-u_{1}(s)-w_{1}(s)$ will increase to 0 .

Upon touching $1-u-w=0, v<0, z<0$, the trajectory $\mathbf{y}_{1}(s)$ enters $R$. This is a contradiction.

If $w_{1}(s)=0$, let $s_{1}$ be the first such time. Then $z_{1}\left(s_{1}\right) \leqslant 0$. Since $\mathbf{y}_{1}(s) \notin \mathscr{V}$, $z_{1}\left(s_{1}\right)<0$. Since $\mathbf{y}_{1}\left(s_{1}\right) \notin Q, u_{1}\left(s_{1}\right)<\beta$. Since $\mathbf{y}_{1}\left(s_{1}\right) \notin R, v_{1}\left(s_{1}\right)>0$. Then since $z^{\prime}=c z-\alpha w(u-\beta)<0, z_{1}(s)$ is decreasing from $z_{1}\left(s_{1}\right)<0$ and $w_{1}(s)$ is decreasing from 0 . Therefore the trajectory remains with $u<\beta, v>0, w>0$ until the trajectory crosses the line $u=\beta$ and, of course, at this crossing, $z_{1}(s)<0$. Thus the solution enters $Q$, a contradiction. Therefore $w_{1}(s)>0$ for all $s$.

LEMMA 14. The coordinate functions $u_{1}(s)$ and $w_{1}(s)$ are bounded above by $1, L$, respectively, where $L$ is a positive constant.

Proof. We have $w_{1}(s)>0$ and $y_{1}(s)$ cannot enter the region $S$, so $u_{1}(s)$ is bounded above by 1 .

Suppose $u_{1}(s)>\beta$ for all $s$ and $w_{1}(s)$ is unbounded. We may as well assume $w_{1}(s)>1-\beta$; then, since $\mathrm{y}_{1}(s)$ does not enter $S, v_{1}(s)<0$. If $u_{1}(s)>\beta, v_{1}(s)<0$, and $w_{1}(s)$ increases without bound, then the proof of part 3 of Lemma 7 provides a contradiction. Thus there is a bound $L$ on $w_{1}(s)$ when $u_{1}(s)>\beta$.

Suppose $s_{1}$ is the first time $w_{1}(s)$ exceeds $L$ with $u_{1}(s) \leqslant \beta$. Then since $\mathbf{y}_{1}(s)$ does not enter $P$, we must have $z_{1}(s)<0$. But then $w_{1}(s)$ must have exceeded the value $L$ at some previous time, a contradiction to the choice of $s_{1}$.

This establishes the bounds on $w_{1}(s)$.

LEMMA 15. Let

$$
\begin{aligned}
\mathscr{D}=\{(u, v, w, z) \mid 0<u< & 1,0<w<L, \\
& -(\alpha \beta / c) w<z<d w,-[(L+1) / c] u<v<(2 / c) u\},
\end{aligned}
$$

where $d>c / 2+\left(\sqrt{c^{2}+4 \alpha \beta}\right) / 2$. Then $\mathbf{y}_{1}(s)$ remains in $\mathscr{D}$ for all $s$.

Proof. The bounds on $u_{1}, w_{1}$ have been established in the previous two lemmas.

Suppose there were an $s_{1}$ such that $z_{1}\left(s_{1}\right)<-(\alpha \beta / c) w_{1}\left(s_{1}\right)<0$. If there were an $s_{2}>s_{1}$ such that $z_{1}\left(s_{2}\right)=-(\alpha \beta / c) w_{1}\left(s_{2}\right)$ then $z_{1}^{\prime}\left(s_{2}\right)+(\alpha \beta / c) w_{1}^{\prime}\left(s_{2}\right) \geqslant 0$. Substitution from (5) yields

$$
-\alpha w u-(\alpha \beta / c)^{2} w<0 \quad \text { at } s=s_{2} .
$$


This is impossible, so if $z_{1}\left(s_{1}\right)<-(\alpha \beta / c) w\left(s_{1}\right)$ the inequality continues to hold for $s>s_{1}$. Then for $s>s_{1}, z_{1}^{\prime}=c z-\alpha w_{1}\left(u_{1}-\beta\right)<-\alpha w_{1} u_{1}<0$. Then $z_{1}(s)<z_{1}\left(s_{1}\right)$ for $s>s_{1}$. Therefore $w_{1}^{\prime}(s)=z_{1}(s)$ is strictly negative and bounded away from zero by $z_{1}\left(s_{1}\right)$. Therefore $w_{1}(s)<0$ for some finite $s$, a contradiction. Next, notice that a trajectory starting on $\Sigma$ approaches $(1,0,0,0)$ tangent to $x_{2}$ or $x_{3}$. Points on $x_{2}$ or $x_{3}$ have $z=\lambda_{2} w$ or $z=\lambda_{3} w$. Since $\lambda_{2}, \lambda_{3}<d$, Lemma 3 shows $z_{1}(0)<d w_{1}(0)$ by resetting the initial time if necessary. Let $s=\inf \left\{s>0 \mid z_{1}(s) \geqslant d w_{1}(s)\right\}$. Then $z_{1}\left(s_{1}\right)=d w_{1}\left(s_{1}\right)$ and $z_{1}(s)<d w_{1}(s)$ for $0 \leqslant s<s_{1}$. Therefore $z^{\prime}\left(s_{1}\right)-d w^{\prime}\left(s_{1}\right) \geqslant 0$. Substituting the expressions for $z^{\prime}$ and $w^{\prime}$,

$$
c z-\alpha w(u-\alpha \beta)-d z \geqslant 0 \text { at } s=s_{1} .
$$

Then using $z=d w$, we obtain

$$
\left[-d^{2}+c d-\alpha(u-\beta)\right] w \geqslant 0 .
$$

But the choice of $d$ implies $-d^{2}+c d+\alpha \beta<0$. Therefore $\left[-d^{2}+c d+\alpha \beta\right] w-$ $\alpha u w<0$ at $s=s_{1}$. This contradiction shows that $z_{1}(s)<d w_{1}(s)$ for $s$ such that $w_{1}(s)>0$.

Suppose there were some first time $s_{1}$ when $v=-[(L+1) / c] u_{1}\left(s_{1}\right)$. Then

$$
v_{1}^{\prime}\left(s_{1}\right)=-(1 / D)(L+1) u_{1}\left(s_{1}\right)-(1 / D) u_{1}\left(s_{1}\right)\left(1-u_{1}\left(s_{1}\right)-w_{1}\left(s_{1}\right)\right) \text {. }
$$

Since $1-u_{1}\left(s_{1}\right)-w_{1}\left(s_{1}\right)>-L$, then $v_{1}^{\prime}\left(s_{1}\right)<-(1 / D) u_{1}\left(s_{1}\right)<0$. Thus $v_{1}(s)$ is decreasing from $v_{1}\left(s_{1}\right)$ so $u_{1}^{\prime}(s)=v_{1}(s)<v_{1}\left(s_{1}\right)<0$ for $s>s_{1}$. Therefore $u_{1}(s)$ decreases past $\boldsymbol{u}=0$, which contradicts Lemma 13 .

Suppose there were some first time $s_{1}$ when $v_{1}\left(s_{1}\right)=(2 / c) u_{1}\left(s_{1}\right)$. Then

$$
v_{1}^{\prime}\left(s_{1}\right)=(2 / D) u_{1}\left(s_{1}\right)-(1 / D) u_{1}\left(s_{1}\right)\left(1-u_{1}\left(s_{1}\right)-w_{1}\left(s_{1}\right)\right) \text {. }
$$

Since $1-u_{1}\left(s_{1}\right)-w_{1}\left(s_{1}\right)<1, v_{1}^{\prime}\left(s_{1}\right) \geqslant(1 / D) u_{1}\left(s_{1}\right)>0$. Therefore $v_{1}(s)$ is increasing from $v_{1}\left(s_{1}\right)$ so $u^{\prime}(s)=v(s)>v\left(s_{1}\right)>0$ for $s>s_{1}$. Then $u(s)$ increases beyond $u=1$, a contradiction to Lemma 14 .

Therefore the solution is bounded within the set $\mathscr{D}$. This concludes the lemma.

Consider the function

$$
\begin{aligned}
V(u, v, w, z)= & \alpha[c(u-\beta)-D v]+\alpha \beta[D(v / u)-c \log (u / \beta)] \\
& +[c(w-(1-\beta))-z]+(1-\beta)[z / w-c \log (w /(1-\beta))] .
\end{aligned}
$$

Then $V$ is continuous on $\mathscr{D}$. By checking each summand separately, it is easy to show $V$ is bounded below on $\mathscr{D}$. Using the vector field from the right-hand side of (5), it is easy to check that

$$
V^{\prime}=-\alpha(u-\beta)^{2}-\alpha \beta D v^{2} / u^{2}-(1-\beta) z^{2} / w^{2}<0 .
$$

Therefore $V$ is a Liapunov function for (5) on $\mathscr{D}$.

Notice that $V^{\prime}=0$ if and only if $(u, v, w, z)$ is on the segment defined by $u=\beta$, $v=0,0<w<L, z=0$.

If $0<w<1-\beta$ and $(u, v, w, z)$ is on this segment, then

$$
w^{\prime}=z=0, \text { and } w^{\prime \prime}=z^{\prime}=c z-\alpha w(u-\beta)=0 \text {. }
$$


Also

$$
w^{\prime \prime \prime}=z^{\prime \prime}=c z^{\prime}-\alpha z(u-\beta)-\alpha w v=0 .
$$

But

$$
\begin{aligned}
w^{(4)} & =c z^{\prime \prime}-\alpha z^{\prime}(u-\beta)-\alpha z^{\prime} v-\alpha z v-\alpha w v^{\prime} \\
& =-\alpha w((c / D) v-(u / D)(1-u-w))=-\alpha w\left(-\frac{\beta}{D}(1-\beta-w)\right)>0 .
\end{aligned}
$$

Therefore, $w$ has a local minimum and $z$ is increasing from 0 .

Likewise, if $1-\beta<w<L$, then $w^{(4)}<0$, so $w$ has a local maximum and $z$ is decreasing.

Therefore the largest invariant subset of this segment is the single point $(\beta, 0,1-$ $\beta, 0)$. The Invariance Principle applies to show that $\mathbf{y}_{1}(s) \rightarrow(\beta, 0,1-\beta, 0)$ as $s \rightarrow \infty$. This completes the proof of the existence of waves when $c^{2}>4 \alpha(1-\beta)$.

F. The traveling wave front solutions exist if $c^{2}>4 \alpha(1-\beta)$. Such solutions approach $(\beta, 0,1-\beta, 0)$ and therefore lie on the stable manifold of the critical point $(\beta, 0,1-\beta, 0)$. The present section shows that the stable manifold is two dimensional. This allows a classification of the asymptotic behavior of the solutions as monotone or oscillatory depending upon whether the critical point is a node or a spiral point on the stable manifold. The characteristic polynomial of system (3) linearized about $(\beta, 0,1-\beta, 0)$ is

$$
p(\lambda)=\lambda^{2}(\lambda-c)(\lambda-c / D)-(\beta / D) \lambda(\lambda-c)+\alpha \beta(1-\beta) / D .
$$

Using the Hurwitz algorithm as given by [11, p. 190], the matrix always has two eigenvalues with positive real part and two eigenvalues with negative real part. This shows that there is always a 2-dimensional stable manifold and a 2-dimensional unstable manifold at $(\beta, 0,1-\beta, 0)$. By graphing $p(\lambda)$ for several values of $\alpha \geqslant 0$, it is easily seen that there is a critical value $\alpha^{*}=\alpha^{*}(\beta, c, D)$. If $\alpha<\alpha^{*}$, there are two distinct negative real eigenvalues. If $\alpha=\alpha^{*}$, there is a repeated negative real eigenvalue. If $\alpha>\alpha^{*}$, there is a complex conjugate pair of eigenvalues with negative real part.

Therefore, if $\alpha \leqslant \alpha^{*}$, solutions to (4.1) on the stable manifold approach ( $\beta, 0,1-$ $\beta, 0)$ monotonely. If $\alpha>\alpha^{*}$, solutions of 3.1 on the stable manifold will spiral in toward $(\beta, 0,1-\beta, 0)$ with damped oscillations.

Let

$$
p_{1}(\lambda, D)=D P(\lambda)=\lambda^{2}(\lambda-c)(D \lambda-c)-\beta \lambda(\lambda-c)+\alpha \beta(1-\beta) .
$$

Consider

$$
p_{1}(\lambda, 1)=\lambda^{2}(\lambda-c)^{2}-\beta \lambda(\lambda-c)+\alpha \beta(1-\beta) .
$$

By completing the square,

$$
p_{1}(\lambda, 1)=[\lambda(\lambda-c)-\beta / 2]^{2}+\alpha \beta(1-\beta)-\beta^{2} / 4 \text {. }
$$


Then it is clear that $p_{1}(\lambda, 1)$ will have a local minimum when $\lambda^{2}-c \lambda-\beta / 2=0$. That is, when $\lambda_{0}=\left(c \pm \sqrt{c^{2}+2 \beta}\right) / 2$,

$$
p_{1}\left(\lambda_{0}, 1\right)=\alpha \beta(1-\beta)-\beta^{2} / 4 \text {. }
$$

If $4 \alpha<\beta /(1-\beta)$, the local minimum will be negative, therefore $p_{1}(\lambda, 1)$ will have two negative real roots.

Now for $D \leqslant 1$ and $\lambda_{0}=\left(c-\sqrt{c^{2}+2 \beta}\right) / 2$,

$$
D \lambda_{0} \geqslant \lambda_{0}, \text { since } \lambda_{0}<0 \text {. }
$$

Then $\lambda_{0}\left(D \lambda_{0}-c\right) \leqslant \lambda_{0}\left(\lambda_{0}-c\right)$. Next, $\lambda_{0}\left(\lambda_{0}-c\right)=\beta / 2>0$, so

$$
\lambda_{0}^{2}\left(D \lambda_{0}-c\right)\left(\lambda_{0}-c\right) \leqslant \lambda_{0}^{2}\left(\lambda_{0}-c\right)^{2} \text {. }
$$

Therefore,

$$
\begin{aligned}
& \lambda_{0}^{2}\left(D \lambda_{0}-c\right)\left(\lambda_{0}-c\right)-\beta \lambda_{0}\left(\lambda_{0}-c\right)+\alpha \beta(1-\beta) \\
& \quad \leqslant \lambda_{0}^{2}\left(\lambda_{0}-c\right)^{2}-\beta \lambda_{0}\left(\lambda_{0}-c\right)+\alpha \beta(1-\beta)=\alpha \beta(1-\beta)-\beta^{2} / 4 \leqslant 0,
\end{aligned}
$$

provided $4 \alpha \leqslant \beta /(1-\beta)$. Thus, if $D \leqslant 1$ and $4 \alpha \leqslant \beta /(1-\beta)$, we have $p_{1}\left(\lambda_{0}, D\right)$ $<0$, so there are two negative real roots. Therefore $\alpha^{*}>\beta / 4(1-\beta)$.

Appendix I. In §3B we defined blocks $P, Q, R, S$ which were removed from $\mathbf{R}^{4}$ to form the Wazewski set

$$
W=\mathbf{R}^{4} \backslash(P \cup Q \cup R \cup S) .
$$

We also described the immediate exit set

$$
W^{-}=\partial W \backslash\left(\{(\beta, 0,1-\beta, 0)\} \cup J_{1} \cup J_{2}\right),
$$

where $J_{1}, J_{2}$ were described in $\S 3 \mathrm{~B}$. The details of the proof that $W^{-}$is the set described above are tedious. As an example, we only examine the portion $\partial R \backslash P$ of $\partial W$. This will show why the set $J_{2}$ must be excluded from $\partial W$ to obtain $W^{-}$. The complete proof can be found in [7].

1. $u=\beta, w<1-\beta, v<0$. Then $u^{\prime}=v<0$ and the solution enters $R$ regardless of the sign of $z$.

2. $u=\beta, w=1-\beta, v<0$.

(a) $z<0$. Then $u^{\prime}=v<0, w^{\prime}=z<0$, and the trajectory enters $R$.

(b) $z=0$. Then $(1-u-w)^{\prime}=-(v+z)>0$ and $u^{\prime}=v<0$, so $(1-u-w)$

is increasing and $u$ is decreasing. Thus the trajectory enters $R$.

(c) $z>0$. Then $u^{\prime}=v<0, w^{\prime}=z>0$, and the trajectory enters $P$.

3. $u=\beta, w=1-\beta, v=0$.

(a) $z<0$. Then $(1-u-w)^{\prime}=-(v+z)>0$. Also

$$
u^{\prime \prime}=v^{\prime}=(c / D) v-(1 / D) u(1-u-w)=0
$$

and

$$
u^{\prime \prime \prime}=v^{\prime \prime}=(c / D) v^{\prime}-(1 / D) v(1-u-w)-(1 / D) u(-v-z)<0 .
$$

Thus $(1-u-w)$ is increasing, $u$ is decreasing, and $v$ has a maximum. The trajectory enters $R$.

(b) $z=0$. This is a singular point and is not in the immediate exit set. 
(c) $z>0$. Then $u^{\prime \prime}=v^{\prime}=0$ and $u^{\prime \prime \prime}=v^{\prime \prime}>0$. Therefore $u$ is increasing, $v$ has a minimum, and $w^{\prime}=z>0$, so $w$ is increasing. Therefore, the trajectory enters $S$.

4. $u=\beta, w<1-\beta, v=0$. Then

$$
u^{\prime \prime}=v^{\prime}=(c / D) v-(1 / D) u(1-u-w)<0,
$$

so $u$ has a maximum and $v$ is decreasing. The trajectory enters $R$ regardless of the sign of $z$.

5. $u<\beta, 1-u-w=0, v<0$.

(a) $z<0$. Then $(1-u-w)^{\prime}=-(v+z)>0$, so $(1-u-w)$ is increasing and the trajectory enters $R$.

(b) $z=0$. Then $(1-u-w)^{\prime}=-(v+z)>0$, so the trajectory still enters $R$.

(c) $z>0$. These points are in the region $P$ and so are not considered.

6. $0<u<\beta, 1-u-w=0, v=0$.

(a) $z<0$. Then $(1-u-w)^{\prime}=-(v+z)>0$, so $(1-u-w)$ is increasing. Also $u^{\prime \prime}=v^{\prime}=0$, and

$$
u^{\prime \prime \prime}=v^{\prime \prime}=(c / D) v^{\prime}-(1 / D) v(1-u-w)-(1 / D) u(-v-z)<0,
$$

so $u$ is decreasing and $v$ has a maximum, so the trajectory enters $R$.

(b) $z=0$. Then $u<\beta, 1-u-w=0$ implies $w>1-\beta$, and $z^{\prime}>0$, so the trajectory enters $P$.

(c) $z>0$. Then $u<\beta$, and $1-u-w=0$ implies $w>1-\beta$, so these points are in $P$ and are not considered.

7. $u<0,1-u-w=0, v=0$.

(a) $z<0$. Then $u^{\prime \prime}=v^{\prime}=0$, and

$$
u^{\prime \prime \prime}=v^{\prime \prime}=(c / D) v-(1 / D) v(1-u-w)-(1 / D) u(-v-z)>0,
$$

so $u$ is increasing and $v$ has a minimum. These points are not in $W^{-}$. This is included in the first portion of $J_{2}$.

(b) $z=0$. Then $u<0,1-u-w=0$ implies that $w>1>1-\beta$, so $z^{\prime}>0$. The trajectory immediately exits into $P$.

(c) $z>0$. These points are in $P$ and so are not considered.

8. $0<u<\beta, 1-u-w>0, w>1-\beta, v=0$.

(a) $z<0$. Then $v^{\prime}=(c / D) v-(1 / D) u(1-u-w)<0$ and the trajectory enters $R$ regardless of $z$.

(b) $z=0$. Then $v^{\prime}<0$ and the trajectory enters $R$ regardless of $z$.

(c) $z>0$. These points are in $P$ and are not considered.

9. $0<u<\beta, 1-u-w>0, w<1-\beta$ and $v=0$. Then $v^{\prime}<0$ and the trajectories enter $R$ regardless of the sign of $z$.

10. $u<0,1-u-w>0, w \geqslant 1-\beta, v=0$.

(a) $z<0$. Then $v^{\prime}>0$ and the trajectory does not enter either $P$ or $R$ immediately. This is included in the first and second portions of $J_{2}$.

(b) $z=0$. Then $z^{\prime}>0$ and the trajectory enters $P$.

(c) $z>0$. These points are in $P$ and not considered. 


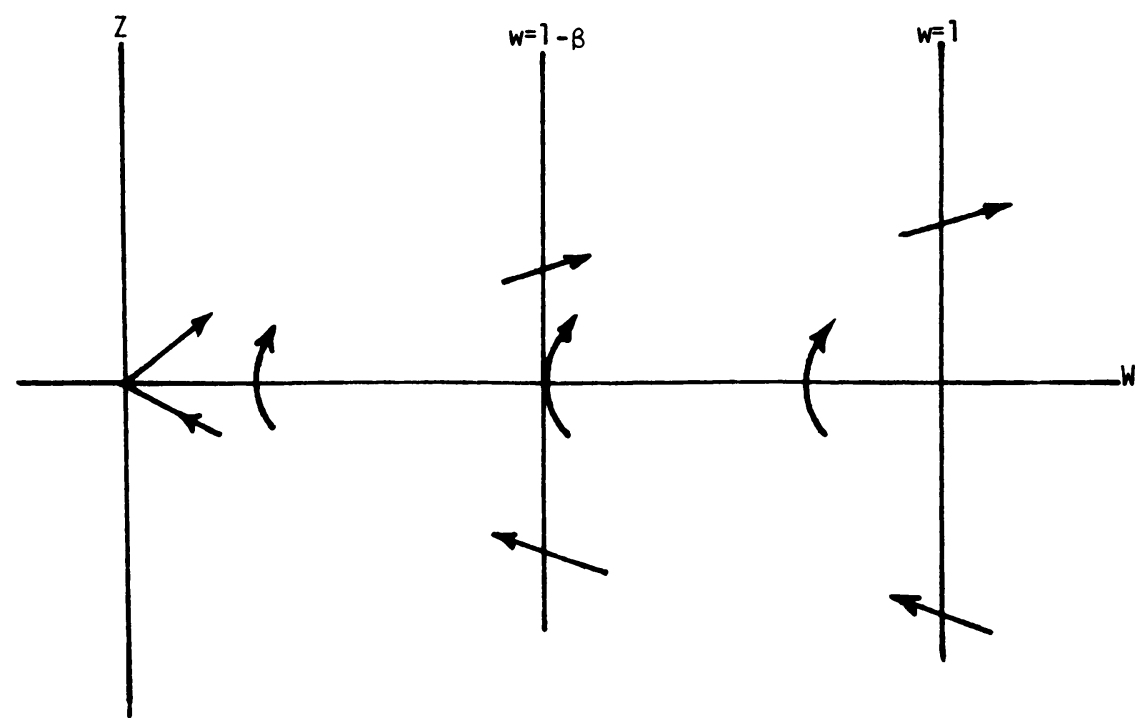

FIGURE 10. Phase plane diagram for exit set analysis.

11. $u<0,1-u-w>0, w<1-\beta, v=0$. Then $v^{\prime}>0$ and the trajectories do not immediately exit into either $P$ or $R$ regardless of the sign of $z$. This is included in the third portion of $J_{2}$.

12. $u=0,1-u-w>0, v=0$.

These points lie on the invariant manifold determined by $u=0, v=0$. The trajectories are solutions of the equation $w^{\prime}=z, z^{\prime}=c z+\alpha \beta w$. The phase diagram of this equation can then determine which points immediately exit $W$. See Figure 10 .

1. $u=0,1-u-w=0, v=0$. Then $w=1>1-\beta$. If $z<0$ these points do not immediately exit into either $P$ or $R$ but remain with $u=v=0, w<1$ for some time. If $z=0$ then the trajectory immediately exits into $P$. If $z>0$ these points are in $P$ and are not considered.

2. $u=0,1-u-w>0, v=0$. Then if $1-\beta<w<1$ and $z<0$, these points do not immediately exit into either $P$ or $R$. If $z=0$ then the trajectories immediately enter $P$. If $z>0$ these points are in $P$ and are not considered.

3. $u=0,1-u-w>0, v=0, w \leqslant 1-\beta$. If $z<0$ these points do not immediately exit into either $P$ or $R$ but remain with $u=v=0, w<1-\beta$ for some time. If $w=1-\beta$ and $z \geqslant 0$, the trajectory immediately exits into $P$. If $w<1-\beta$ and $z \geqslant 0$ the trajectory does not immediately exit into either $P$ or $R$ but remains with $u=v=0, w<1-\beta$ for some time. These three are summarized in the second and third portions of $J_{2}$.

Appendix II. The purpose of this appendix is to show that the set $V$ defined in §3E, namely $V=(\bar{P} \cup \bar{Q} \cup \bar{R} \cup \bar{S}) \backslash\{(\beta, 0,1-\beta, 0)\}$, is not simply connected. The way we accomplish this may be roughly described as follows. We define a homotopy on all of $\mathbf{R}^{4}$, which is then applied to the set $V$. The homotopy on all of $\mathbf{R}^{4}$ 
is most easily described in terms of "cylindrical coordinates" for $\mathbf{R}^{4}$; that is, a coordinate system in which one of the coordinates, $w$, is Cartesian and the remaining three, $u, v$, and $z$, are in terms of 3-dimensional spherical coordinates. This homotopy is constant along the cylindrical axis of this description of $\mathbf{R}^{4}$ and radial in the spherical coordinates. This makes it simple to describe the homotopy because we need only describe the homotopy in terms of the two angular variables of spherical coordinates. This is easily done by describing a homotopy on the surface of the unit sphere in $\mathbf{R}^{3}$. This last homotopy is not simple to write in terms of a function, although it is certainly possible, and we content ourselves with a geometric description. Having done this, we have the desired function, which we will call $h$ : $\mathbf{R}^{4} \times[0,1] \rightarrow \mathbf{R}^{4}$. Because of the cylindrical coordinate description of $h$, it is particularly simple to describe its action. In fact, the homotopy $h$ is constant in the cylindrical coordinate and, in the remaining three coordinates, it "rotates out" the second component of $\mathbf{R}^{4}$, in the original Cartesian description of $\mathbf{R}^{4}$. So the end result of the homotopy lies in the subspace $(u, 0, w, z)$; that is, essentially in $\mathbf{R}^{3}$. Next, we apply the function $h$ to the set $V$ and use this simple description of the action to describe a retraction of $V$ to a subset of $V$ called $A$. It is easily seen that $A$ is not simply connected. Because the retraction of $V$ is not simply connected, neither is $V$ and so we are done.

To be precise, we need a definition from algebraic topology (see [4, p. 39, 5.1; or 2 . p. 25]).

Definition. A subset $A$ of a topological space $X$ is a strong deformation retract of $X$ if there is a continuous function $h: X \times[0,1] \rightarrow X$ such that:

1. $h(x, 0)=x$ for all $x \in X$,

2. $h(x, 1) \in A$ for all $x \in X$,

3. $h(x, t)=x$ for all $x \in A$, for all $t \in[0,1]$.

A subset $A$ of a space $X$ which is a strong deformation retract of $X$ is of the same homotopy type as $X$. This follows because the retraction $h(x, 1)$ of $X$ to $A$ is homotopic to the identity on $X$.

We now describe a strong deformation retraction of $V$ to a subset $A$ of $V$. $A$ is, in turn, equivalent to $\mathbf{R}^{3}$ with a line removed. That is, $A$ is not simply connected and since $V$ has the same homotopy type as $A, V$ is not simply connected. Since $V$ is a subset of $\mathbf{R}^{4}$ we will describe a function $h$ on $\mathbf{R}^{4} \times[0,1]$ and then apply this function to $V$. To facilitate the description of $h$, we describe $\mathbf{R}^{4}$ in terms of a cylindrical coordinate system centered at $u=\beta, v=0, w=1-\beta, z=0$. The 3-dimensional $u-v-z$ subspace is in terms of a spherical coordinate system centered at $u=\beta, v=0$, $z=0$. The $w$ subspace is described in terms of a Cartesian coordinate line centered at $w=1-\beta$. Thus the line parallel to the $w$ coordinate axis through the point $u=\beta, v=0, w=1-\beta, z=0$ is the cylindrical axis of this description of $\mathbf{R}^{4}$. The description of $\mathbf{R}^{4}$ is then:

$$
\begin{gathered}
u-\beta=r \cos \theta \sin \phi, \quad v=r \sin \theta \sin \phi, \\
w-(1-\beta)=x, \quad z=r \cos \phi
\end{gathered}
$$


where $0 \leqslant r<\infty, 0 \leqslant \theta<2 \pi, 0 \leqslant \phi \leqslant \pi,-\infty<x<+\infty$. We now define $h$ : $\mathbf{R}^{4} \times[0,1] \rightarrow \mathbf{R}^{4}$ by

$$
h(r, \theta, \phi, x, t)=\left\{\begin{array}{l}
r \cos \Theta(t) \sin \Phi(t), \\
r \sin \Theta(t) \sin \Phi(t), \\
x, \\
r \cos \Phi(t) .
\end{array}\right.
$$

This means that to completely describe the function $h$, we must describe the homotopies $\Theta(t)$ and $\Phi(t)$ in the angular variables. A convenient way to do this is to describe a sequence of homotopies of the unit sphere in $\mathbf{R}^{3}$ and chain these homotopies together into a single homotopy. Although it would be possible to write analytic expressions for each of the homotopies we are about to describe, these would not be simple, and it does not appear that they would add significant understanding to the geometric description.

The ultimate object of this sequence of homotopies is to expand the spherical triangular regions

$$
T_{1}=\{(\theta, \phi) \mid 3 \pi / 2<\theta<2 \pi, 0<\phi<\pi / 2\},
$$

and

$$
T_{2}=\{(\theta, \phi) \mid \pi / 2<\theta<\pi, \pi / 2<\phi<\pi\}
$$

to fill the right and left hemispheres, respectively, of the sphere. See Figure 11. In the original Cartesian coordinates, this has the effect of expanding the octants $\{(u, v, w, z) \mid u>\beta, v<0, z>0\}$ and $\{(u, v, w, z) \mid u<\beta, v>0, z<0\}$ to fill the half spaces $\{(u, v, w, z) \mid v<0\}$ and $\{(u, v, w, z) \mid v>0\}$, respectively. The first of the sequence of homotopies is to rotate the unit sphere in 3-dimensional space so the open spherical triangular patches $T_{1}, T_{2}$ are centered at the north and south poles of the sphere. This can clearly be done continuously in $t$, starting from the identity mapping of the sphere. The next homotopy is to continuously expand the patches $T_{1}, T_{2}$ to fill the northern and southern hemispheres, keeping the equator fixed, and, in the process, squeezing the region between the patches into the equator. This can be done by expanding the spherical annular regions between the boundaries of the triangular regions $T_{1}, T_{2}$ and a neighborhood of the pole, leaving the neighborhood of the pole and the equator unchanged. This also can be done continuously in $t$, starting from the identity mapping of the sphere. This homotopy can also be viewed in the more familiar context of the plane by stereographically mapping the sphere to the plane. Then the spherical triangular patch $T_{1}$ surrounding the north pole is mapped to the exterior of a triangle-like region in the plane centered at the origin. The triangular patch $T_{2}$ surrounding the south pole is mapped to a triangle-like patch centered at the origin inside the unit circle in the plane. The homotopy then shrinks the annular region between these patches in the plane to the unit circle in the plane and expands the stereographic images of $T_{1}$ and $T_{2}$ to the exterior of the unit 
circle and the interior of the unit circle respectively. The last homotopy of the unit sphere is simply to rotate the sphere so that the equator becomes the circle $\theta=0 \cup \theta=\pi$. This also can be done continuously, starting from the identity mapping, just as for the first homotopy. See Figure 11.

Notice that, as a function of $t, h$ is constant in $x$ or, equivalently, in $w$. This means the action of $h$ on $\mathbf{R}^{4}$ occurs only in the $u-v-z$ subspace. This makes it easy to describe the action of $h$ on the set $V$.

The function $h$ is now restricted to $V$ to yield a strong deformation retraction. The subset $A$ into which the space $V$ is transformed is the set $V \cap\{(u, v, w, z) \mid v=0\}$. Thus for each fixed $w$, the deformation $h$ "folds out" any nonzero $v$ coordinate of the set $V$ into a subset $A$ lying entirely in the 3-dimensional subspace $\{(u, v, w, z) \mid v$ $=0\}$. The easiest way to view the action of $h$ on $V$ is to take a cross-section of $V$ at a

$\phi$

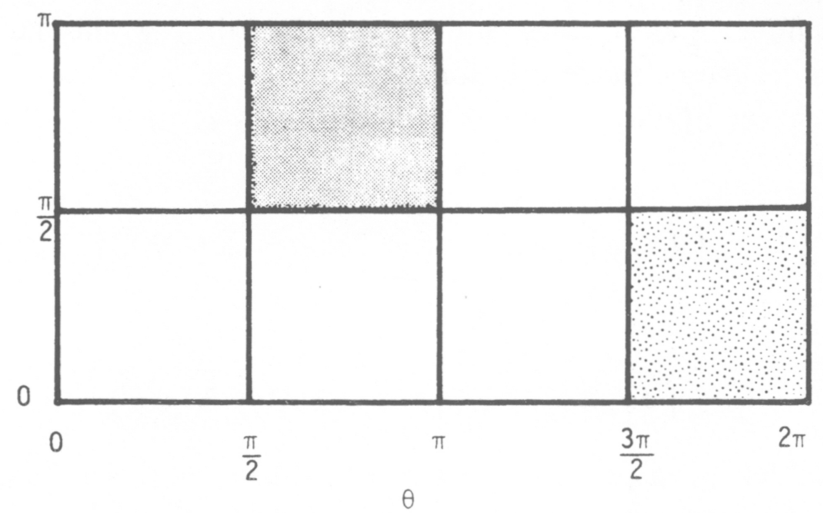

$\phi$
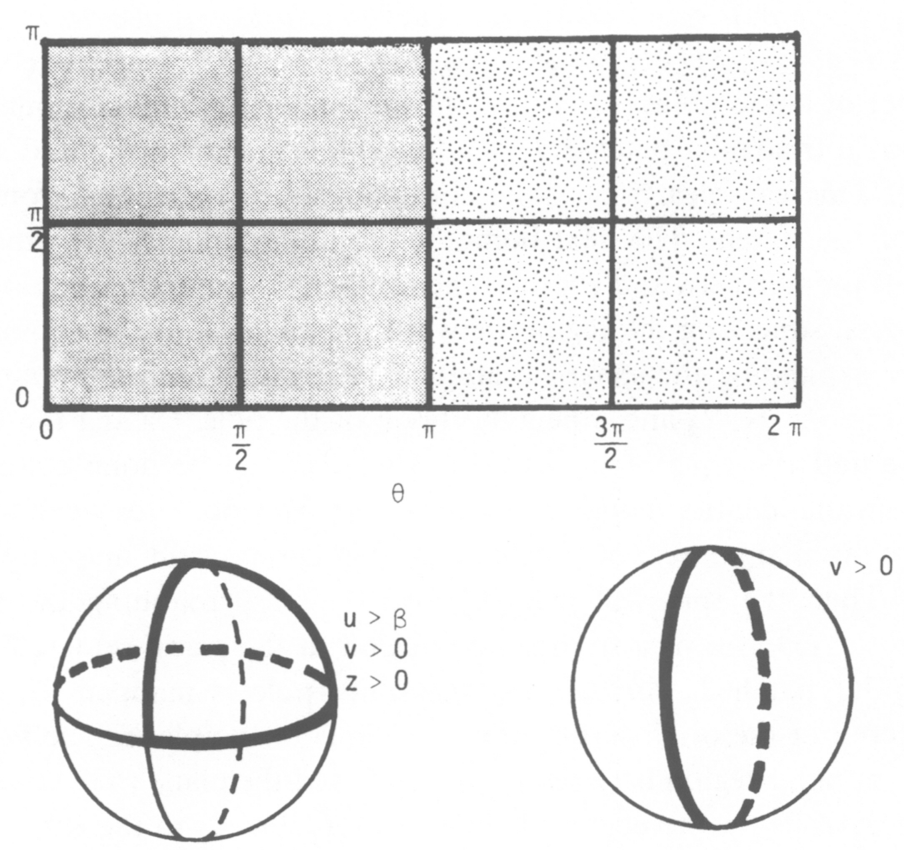

FIGURE 11. Diagram of the strong deformation retraction $h(r, \theta, \phi, x, t)$. 
fixed value of $w$, e.g. $w>1-\beta, w=1-\beta$, and $w<1-\beta$. Then observe the action of $h$ on the 3-dimensional cross-section of $V$ in the $u-v-z$ subspace at the fixed value of $w$. The result in each cross-section lies in the 2-dimensional subspace defined by $v=0$.

Finally, synthesize in $w$ again the results of this section. Thus, the result synthesized back in $w$ is 3-dimensional and easily visualized. See Figures 12-19 for diagrams of this process. Figures 18 and 19 are diagrams of the synthesis back in $w$.

The result of the deformation $h$ on the set $V$ is pictured in Figure 19. The resulting $A$ is a copy of $\mathbf{R}^{3}$ in the $v=0$ subspace of $\mathbf{R}^{4}$ with the closed semi-infinite wedges $U_{1}$ and $U_{2}$ removed, where

$$
\begin{aligned}
& U_{1}=\{(u, v, w, z) \mid v=0, \beta \leqslant u \leqslant 1-w, z \geqslant 0\}, \\
& U_{2}=\{(u, v, w, z) \mid v=0,1-w \leqslant u \leqslant \beta, z \leqslant 0\} .
\end{aligned}
$$

Notice that the point $(\beta, 0,1-\beta, 0)$, where the two wedges meet corner to corner, is removed.

This set $A$ is easily seen to be not simply connected. A loop in $A$ surrounding the removed point cannot be contracted to a point in $A$. Alternatively, the removed

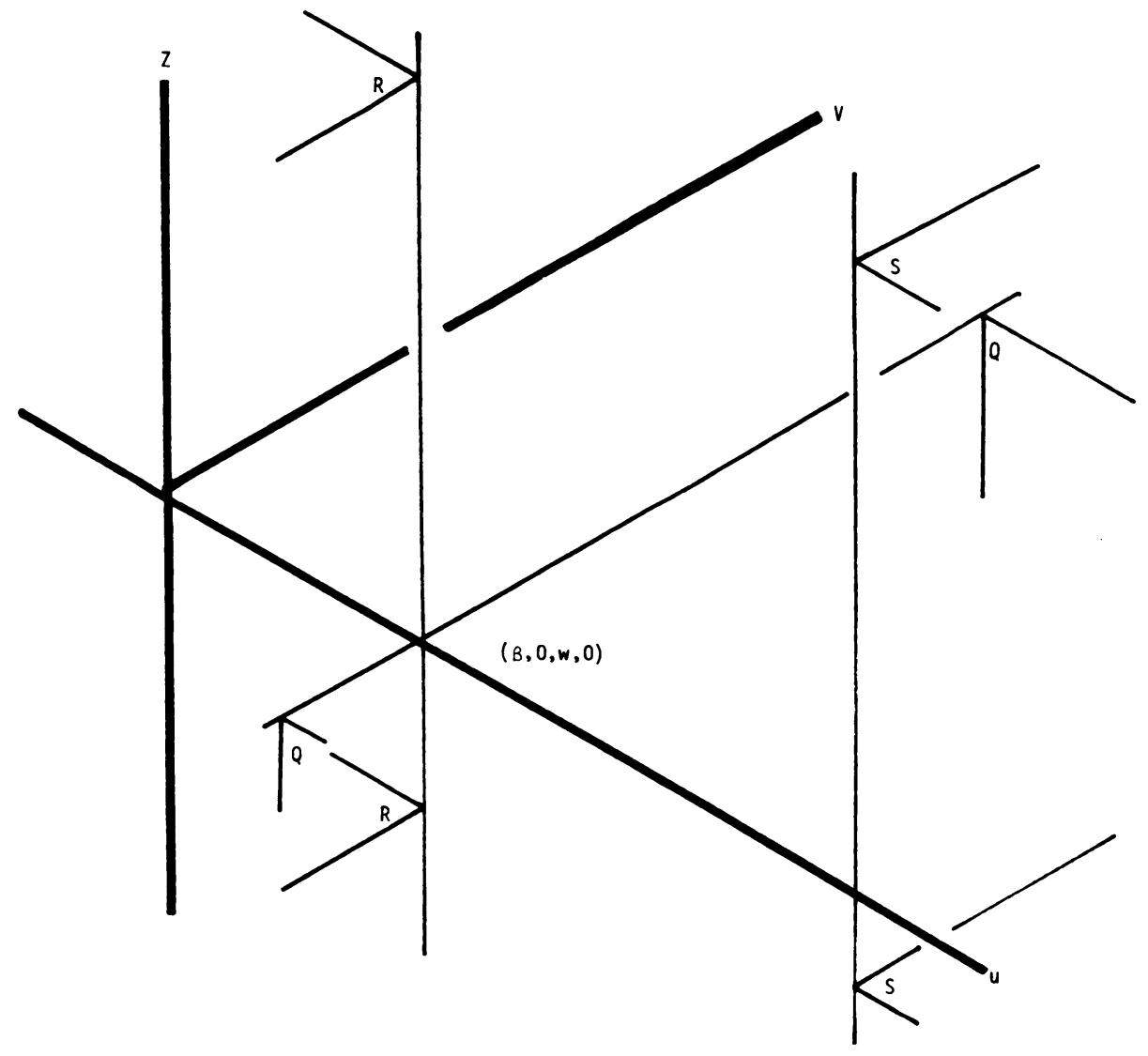

FIGURE 12.

Slice of $V$ in $u-v-z$ space at fixed value of $w<1-\beta$ before retraction. 
semi-infinite wedges $U_{1}$ and $U_{2}$ may be contracted to a pair of half lines meeting at the point $(\beta, 0,1-\beta, 0)$. Thus the set $A$ is homotopically equivalent to $\mathbf{R}^{3}$ with a line removed, which is not simply connected. This finishes the proof.

ACKNOwLEDGEMENT. This paper is a revised portion of the author's Ph.D. thesis completed at the University of Minnesota. The author wishes to thank his thesis adviser Professor D. G. Aronson for his help and encouragement. The author also wishes to thank the reviewer for several helpful suggestions which improved the exposition of the paper.

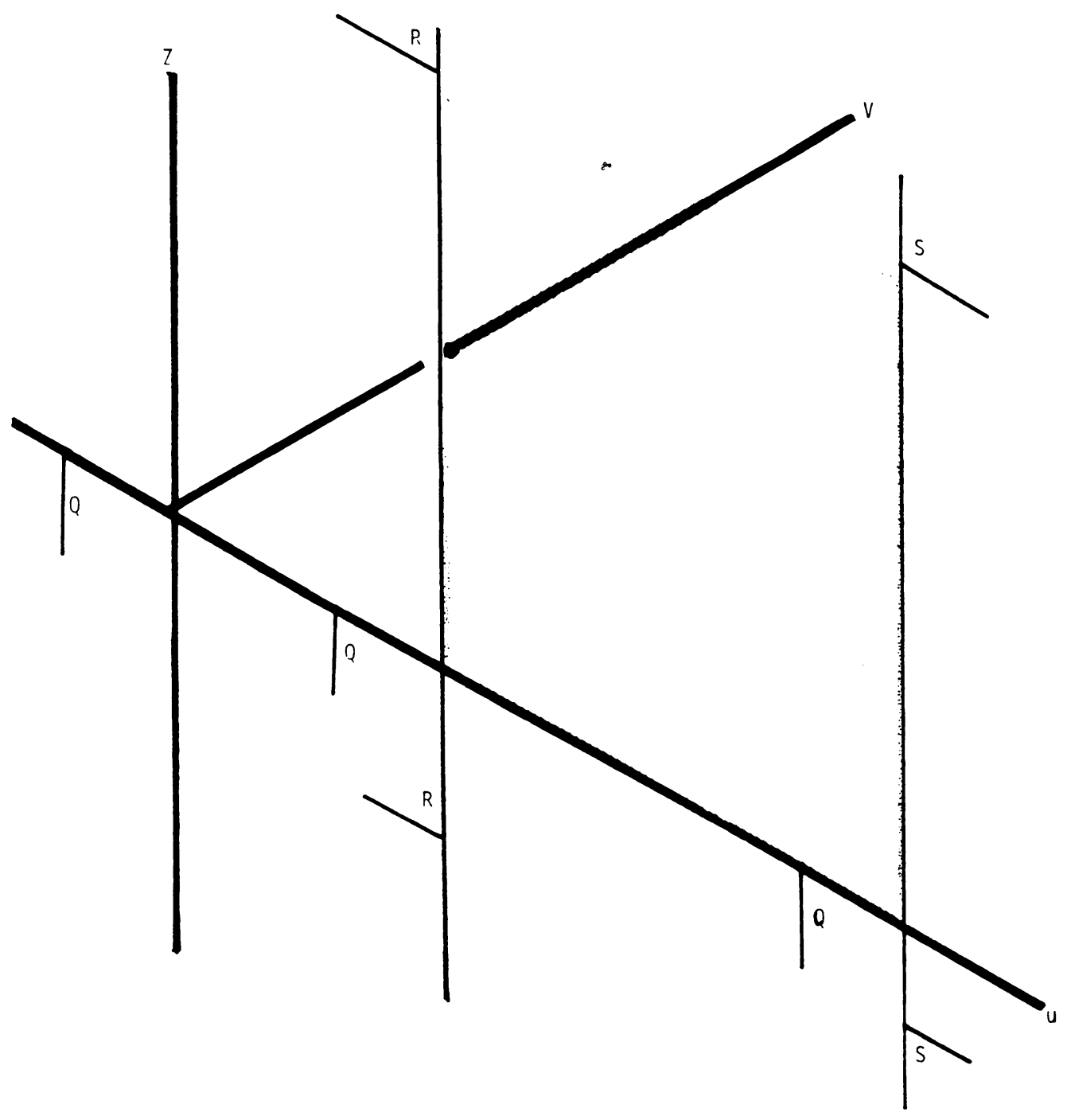

Figure 13.

Slice of $V$ in $u-v-z$ space at fixed value of $w<1-\beta$ after retraction. The shaded region is not in image of $V$ after retraction. 


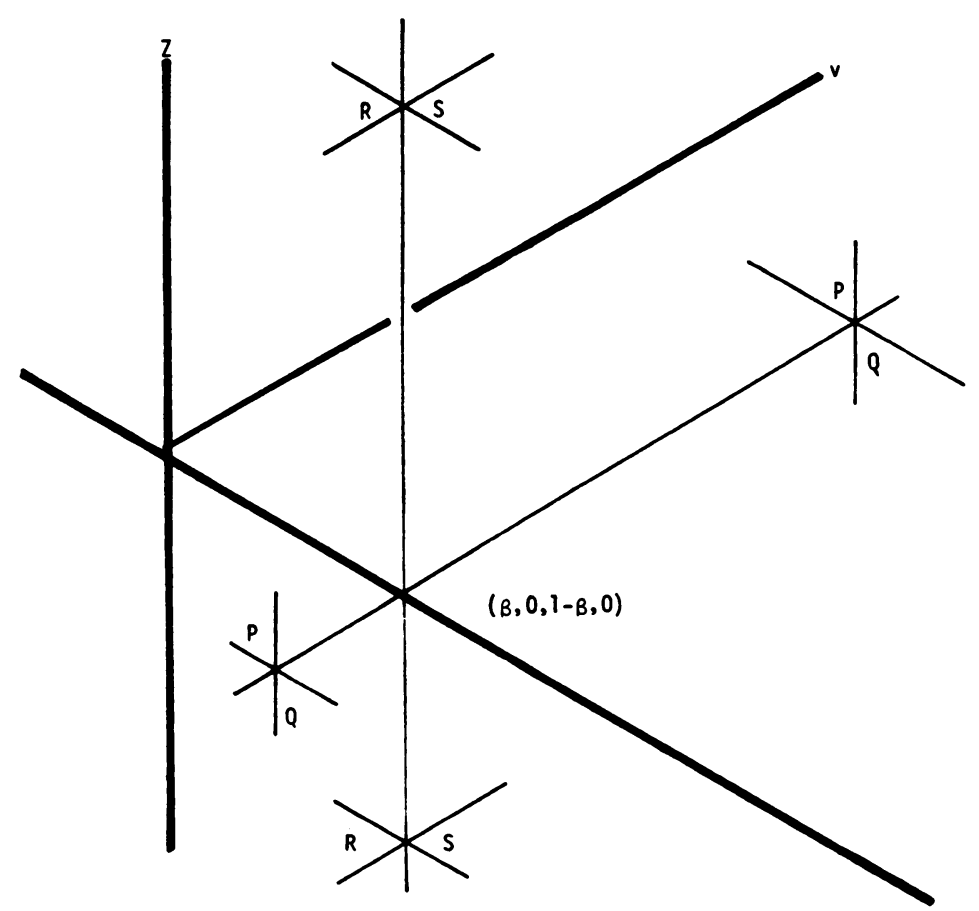

FIgURE 14. Slice of $V$ in $u-v-z$ space at $w=1-\beta$ before retraction.

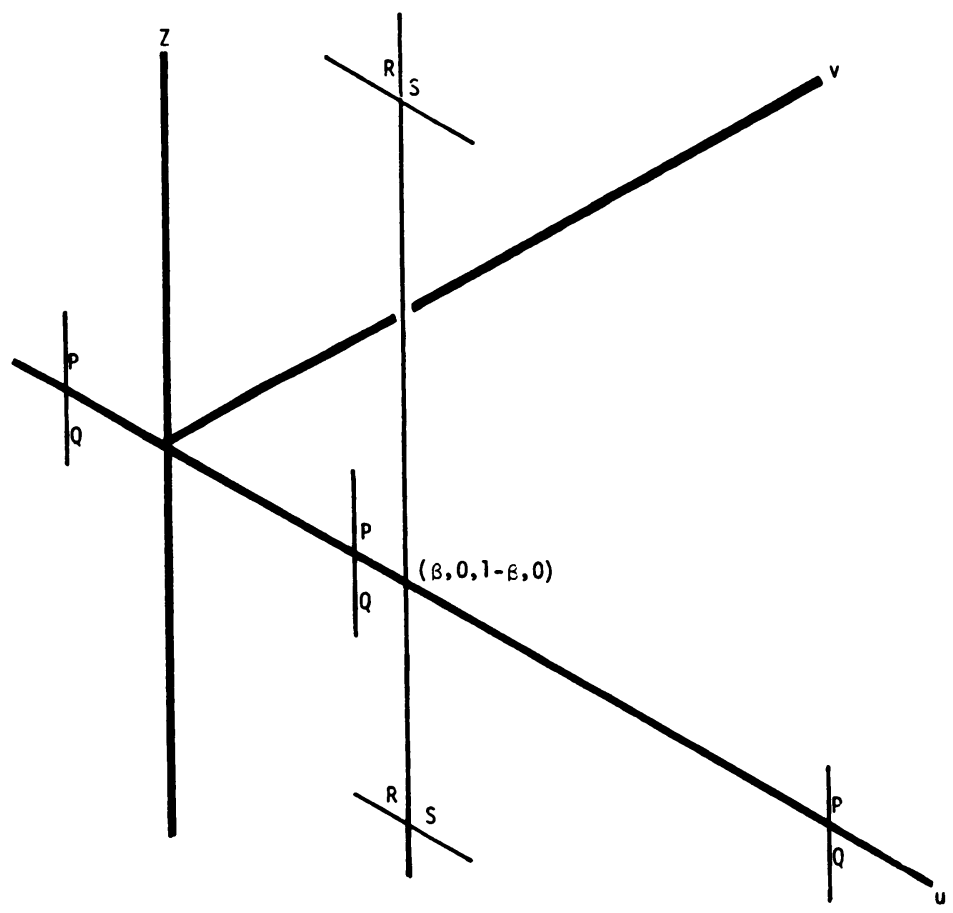

Figure 15.

Slice of $V$ in $u-v-z$ space at $w=1-\beta$. The point $(\beta, 0,1-\beta, 0)$ is not in the image of $V$ after retraction. 


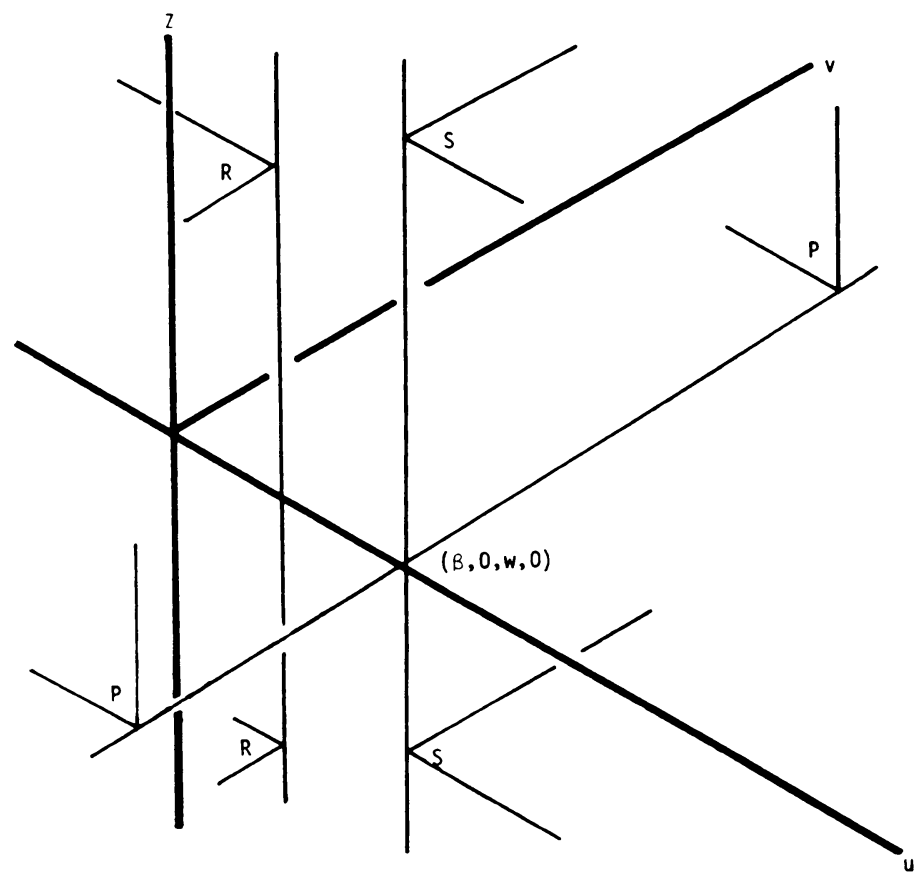

FIGURE 16. Slice of $V$ in $u-v-z$ at fixed value of $w>1-\beta$ before retraction.

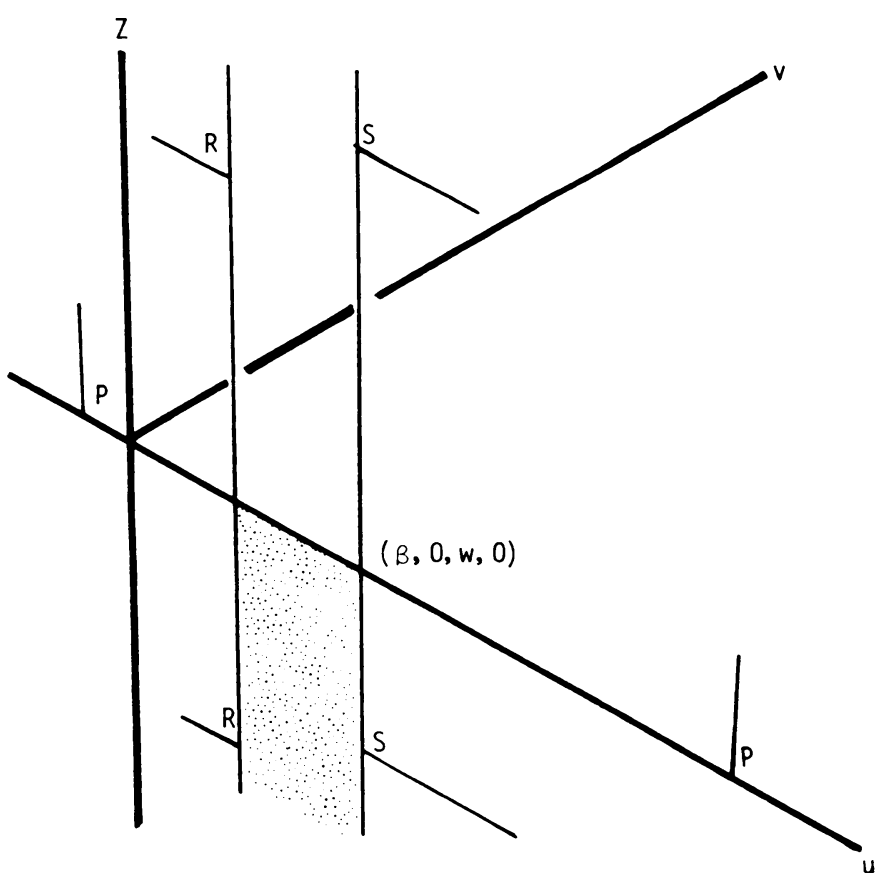

FIGURE 17.

Slice of $V$ in $u-v-z$ space at fixed value of $w>1-\beta$ after retraction. The shaded region is not in the image of $V$ after retraction. 


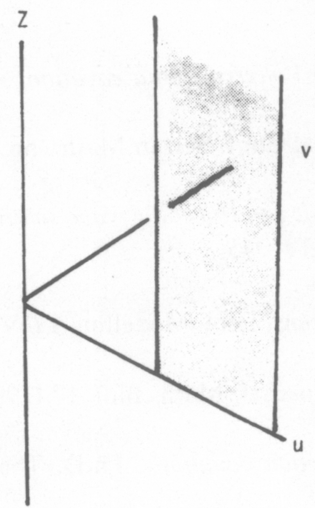

$w<1-\beta$

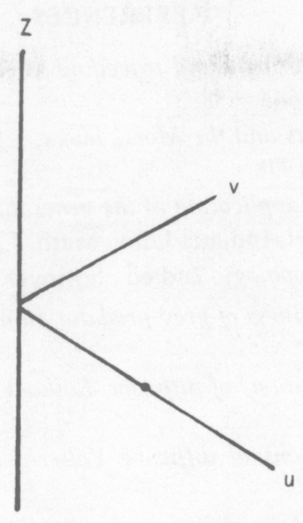

$w=1-6$

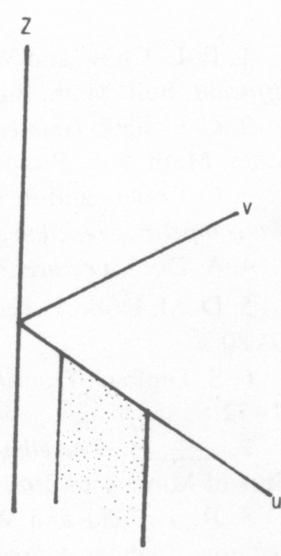

$w=1-$

Figure 18.

Summary of Figures 13, 15, 17 showing the regions which are not in the image of $V$ after retraction.

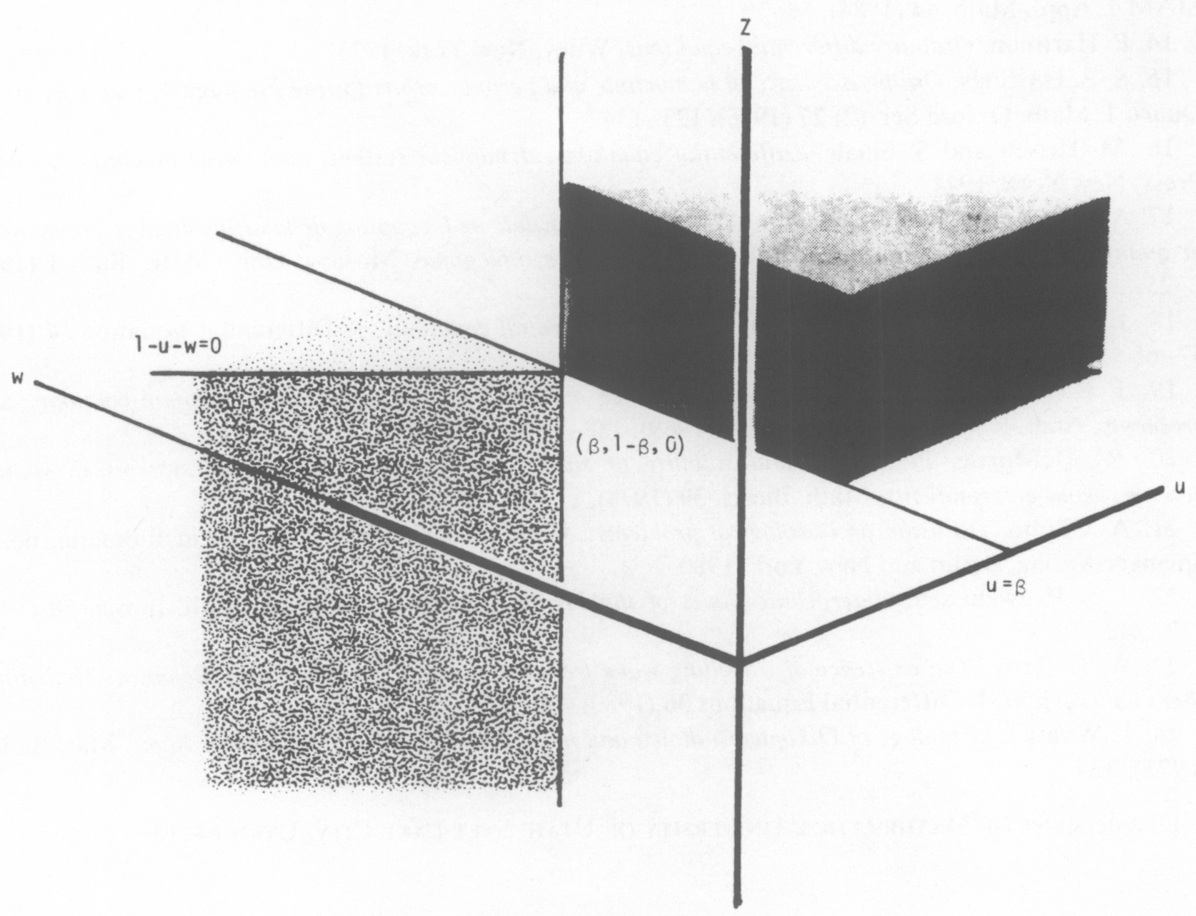

FIGURE 19.

Synthesis in $u-w-z$ space of the regions not in the image of $V$ after retraction. The synthesis of the planes summarized in Figure 18 results in the wedges $\{u>\beta, 1-u-w>0, z>0\}$ and $\{u \leqslant \beta$, $1-u-w \leqslant 0, z \leqslant 0\}$ pictured above. 


\section{REFERENCES}

1. P. L. Chow and W. C. Tam. Periodic and travelling n'ave solutions to Volterra-Lotka equations with diffusion, Bull. Math. Biol. 12 (1976), 643-658.

2. C. Conley, Isolated invariant sets and the Morse index. CBMS Regional Conf. Ser. in Math.. no. 38 . Amer. Math. Soc., Providence, R. I., 1978.

3. C. Conley and R. Gardner, An application of the generalized Morse index to tratelling nate solutions of a competitive reaction diffusion model, Indiana Univ. Math. J., 33 (1984), 319-345.

4. A. Dold. Lectures on algebraic topologi. 2nd ed., Springer, Berlin. 1980.

5. D. M. Dubois, A model of patchiness of prey-predator plankton populations. Ecol. Modelling 1 (1975). 67-80.

6. S. Dunbar, Travelling wave solutions of diffusive Lotka-Volterra equations, J. Math. Biol. 17 (1983). 11-32.

7. Travelling wate solutions of diffusive Volterra-Lotka interaction equations. Ph.D. Thesis. Univ of Minnesota, 1981.

8. R. J. Field and W. C. Troy, The existence of solitan tratelling vate solutions of a model of the Belousot-Zhabotinskii reaction, SIAM J. Appl. Math. 37 (1979), 561-587.

9. P. C. Fife, Mathematical aspects of reacting and diffusing sistems, Lecture Notes in Biomat.. no. 28. Springer-Verlag. Berlin and New York. 1979.

10. R. A. Fisher, The wave of advance of advantageous genes, Ann. Eugenics 7 (1937), 335-369.

11. F. R. Gantmacher, The theon of matrices, Vol. 2. Chelsea, New York, 1964

12. R. Gardner, Existence and stability of tracelling wate solutions of competition models: A degree theoretic approach, J. Differential Equations 44 (1982), 343-364.

13. Existence of travelling wate solutions of predator-prey systems via the connection index.

SIAM J. Appl. Math. 44 (1984), 56-79.

14. P. Hartman, Ordinary differential equations, Wiley, New York. 1973.

15. S. P. Hastings, On the existence of homoclinic and periodic orhits for the Fitzhugh-Nagumo equations, Quart. J. Math. Oxford Ser. (2) 27 (1976), 123-134.

16. M. Hirsch and S. Smale, Differential equations, dinamical sistems and linear algebra. Academic Press, New York, 1974.

17. A. Kolmogorov, I. Petrovsky and N. Piscounov, Étude de l'equation de la diffusion at'ec croissance de la quantité de matière et son application à un probleme hiologique. Moscow Univ. Math. Bull. 1 (1937). $1-25$.

18. J. P. LaSalle, Stability theory for ordinary differential equations, J. Differential Equations 4 (1968). $57-65$.

19. J. B. McLeod and J. Serrin. The existence of similar solutions for some laminar houndan layer problems, Arch. Rational Mech. Anal. 31 (1969), 288-303.

20. R. McMurtie. Persistence and stability of single species and prey-predator systems in spatially heterogeneous eneironments, Math. Biosci. 39 (1978), 11-51.

21. A. Okubo, Diffusion and ecological problems: Mathematical models, Lectures in Biomath., no. 10. Springer-Verlag. Berlin and New York, 1980.

22. J. P. Pauwelussen, Heteroclinic wates of the Fitzhugh-Nagumo equations, Math. Biosci. 58 (1982), 217-242.

23. W. C. Troy, The existence of travelling wave front solutions of a model of the Belousot-Zhabotinskii chemical reaction, J. Differential Equations 36 (1980), 89-98.

24. T. Wyatt. The biologr of Oikopleura dioica and frittilaria horealis in the southern Bight, Mar. Biol. 22 (1973), 137.

Department of Mathematics, University of Utah, Salt Lake City, Utah 84112 